\title{
Surface Pb Nanoparticle Aggregation, Coalescence and Differential Capacitance in a Deep Eutectic Solvent Using a Simultaneous Sample-Rotated Small Angle X-ray Scattering and Electrochemical Methods Approach
}

Joshua A Hammons ${ }^{\mathrm{a}, \mathrm{b} *}$ and Jan Ilavsky ${ }^{\mathrm{a}}$

\begin{abstract}
Nanoparticle electrodeposition is a simple and scalable approach to synthesizing supported nanoparticles. Used with a deep eutectic solvent (DES), surface nanoparticles can be assembled and exhibit unique surface charge separation when the DES is adsorbed on the nanoparticle surface. Key to understanding and controlling the assembly and the capacitance is a thorough understanding of surface particle mobility and charge screening, which requires an in-situ approach. In this study, $\mathrm{Pb}$ particle formation, size, shape and capacitance are resolved in a 1:2 choline $\mathrm{Cl}^{-}$: urea deep eutectic solvent whilst sweeping the cell potential in the range: $0.2 \mathrm{~V}$ to $-1.2 \mathrm{~V}$ (vs. $\mathrm{Ag} / \mathrm{AgCl}$ ). These system parameters were resolved using a complementary suite of sample-rotated small angle X-ray scattering (SR-SAXS) and electrochemical impedance spectroscopy (EIS), which are presented and discussed in detail. This approach is able to show that both particle and ion transport are impeded in the DES, as aggregation occurs over the course of 6 minutes, and dissolved $\mathrm{Pb}$ ions accumulate and remain near the surface after a nucleation pulse is applied. The DES-Pb interactions strongly depend on the cell potential as evidenced by the specific differential capacitance of the $\mathrm{Pb}$ deposit, which has a maximum value of $2.5+/-0.5 \mathrm{~F} \mathrm{~g}^{-1}$ at $-1.0 \mathrm{~V}$ vs. $\mathrm{Ag} / \mathrm{AgCl}$. Altogether, the SR-SAXS-EIS approach is able to characterize the unique nanoparticle capacitance, mobility and ion mobility in a DES and can be used to study a wide range of nanoparticle deposition systems in-situ.

Graphical Abstract
\end{abstract}

Keywords

nanoparticle, synthesis, electrodeposition, deep eutectic solvent, stability, X-ray scattering, SAXS, USAXS, impedance, electrostatic, capacitance, differential capacitance, aggregation, coalescence, $\mathrm{Pb}$ nanoparticles 


\section{Introduction}

Nanoparticle electrodeposition is a simple and cost-effective, bottom-up approach to synthesizing supported nanoparticles[1], which can be used in a variety of nanotechnologies[2]. Owing to its simplicity, electrodeposition is a scalable process that has historically been used in electroplating thin films[1]. Nanoparticle electrodeposition, on the other hand, presents new challenges, particularly pertaining to nanoparticle stability[3], monodispersity[4], and size control[5]. While the latter two concerns can be addressed by tuning the deposition parameters[6-9], the surface particles are mobile[10] and readily aggregate and coalesce[11] into clusters[3, 12]. In general, nanoparticles are stabilized against aggregation by incorporating a stabilizing agent, which adsorbs onto the particle surface to achieve electrostatic, steric or electro-steric mutual repulsion of neighboring particles.[13] This has lead to the pursuit of novel solvents for electrodeposition, which readily adsorb onto particle surfaces as they are synthesized and can dissolve a wide range of metal precursors.

Deep eutectic solvents are a class of tunable, cost-effective and environmentally friendly solutions that are being used in a wide range of electrochemical applications[14]. DESs typically consist of a quaternary ammonium salt and a hydrogen bond donor, at their eutectic composition, and can dissolve a wide range of metal precursors.[15] Mixed together, these two (otherwise solid) components form a clear liquid at room temperature and have been extensively employed since their discovery in 2001.[16] Owing to their unique interactions and propensity to adsorb onto particle surfaces, DESs can both stabilize and assemble nanoparticles during synthesis[17-22]. Therefore, the particle-DES interactions and particle mobility must be understood and are the focus of this study.

\subsection{Particle-DES Interactions}

As the constituent species of a DES are typically too small to achieve steric stabilization, electrostatic stabilization is considered here as the primary stabilization mechanism. This is a process whereby the surface is charged via: ionization of surface groups, ion adsorption, dissolution of ionic solids or isomorphous substitution.[23] Regardless of how the surface is charged, the excess charge must be screened by counter ions. This process results in a local capacitor and an electrostatic surface potential that repels other particles.[23] Both of these 
consequences are desirable for application as electrochemical supercapacitors (EC)[24] and in a broad range of nanotechnologies[2].

The surface charge screening in DESs is in many ways different from that occurring in traditional aqueous solutions or in room temperature ionic liquids.[25-28] While DESs have been effective in producing electrochemical super-capacitors[29-32], the spatial distribution of charge differs significantly from aqueous solution. For example, charged separated perturbation layers have been observed at the nanoparticle surface and extend several nanometers from the surface.[20,25] While this phenomena is likely due to the adsorption of the DES, it is unclear which DES-nanoparticle systems would exhibit this behavior. With the large number of possible combinations and the limited accessibility of synchrotron radiation, the differential capacitance can provide some insight into the interactions between the nanoparticles and DES. For this reason, the differential capacitance was obtained with the X-ray scattering data presented in this study.

Experimentally, the differential capacitances of only a small fraction of DESs have been evaluated. The differential capacitances of these DESs are markedly different from aqueous solutions and depend on both the hydrogen bond donor, as well as the electrode surface.[27, 28] With this study, we will show how the specific differential capacitance in a 1:2 choline $\mathrm{Cl}^{-}$:urea DES exhibits marked variations on the surface of suspended $\mathrm{Pb}$ nanoparticles. Used in combination with the small angle X-ray scattering (SAXS), the specific capacitance can be obtained when multiple cathodic reactions are present, which prevent a mass determination from the faradaic current. This completely in-situ approach is presented and discussed in this paper.

\subsection{Surface Small Angle Scattering}

While all electrochemical investigative techniques are performed in solution, other techniques that probe the physical dimensions of surface phases are often difficult to incorporate in-situ. X-rays, on the other hand, readily penetrate the solution and can be used in-situ. Specifically, SAXS provides an experimenter with the opportunity to resolve nano-scale phases in-situ and over much larger electrode areas compared with modern microscopes. However, SAXS is not as common as electron microscopes, as it not as accessible and can often times be ambiguous. In this paper, the combined sample-rotated 
SAXS /EIS approach is presented and was developed specifically to address data ambiguity. The overall goal of this technique is to provide a comprehensive characterization of the surface and provide the experimenter with better model certainty.

Data ambiguity in small angle scattering (SAS) often arises when the scattering phases are unknown. The combined SR-SAXS/EIS approach reduces this ambiguity by: 1) probing the scattered intensity in three dimensions, 2) providing absolute scattering length densities and volume fractions of scattering phases and 3) correlating the physical dimensions of surface phases with the electrochemical response. In a typical Grazing Transmission SAXS (GTSAXS) experiment, the scattering from surface phases is collected by tilting the sample surface to angles greater than the critical angle.[33] In this way, the dimensions of surface phases, both parallel and perpendicular to the surface, can be extracted from a 2D SAXS image. The difference between a grazing transmission geometry and sample-rotated, used here, is that the incident X-rays are applied to different sample angles that are much larger than the critical angle to avoid the complications of a distorted surface wave. The SR-SAXS approach may be preferred over a grazing incidence (GISAXS) or GTSAXS approach when there is no surface ordering and there are restrictions in cell design. Furthermore, the SR-SAXS can be extended to collect several SAXS patterns at different sample angles, which can remove some model ambiguity when the scattering is anisotropic.[25] In this article, the in-situ geometry, data treatment and analysis are discussed in detail and can be applied to a very broad range of electrochemical systems with uniquely anisotropic surface phases.

\subsection{Particle Mobility and Aggregation}

Surface particle mobility is usually inferred from a post-mortem analysis of a sample. Very few in-situ techniques[10] exist that allow an experimenter to observe the change in surface particle morphology with time. Most often, this is because aggregation occurs on sub-second time scales[3] and it is difficult to probe through the solution. However, synchrotron X-rays can easily penetrate solutions and have been used to observe aggregation and coalescence in-situ in a DES where particle mobility is sufficiently slow[25]. The same SR-SAXS approach[33] is used here simultaneously with electrochemical techniques. We note that in the before mentioned article, the GTSAXS terminology was used. These techniques are complementary, since the data from one technique can be used to extract more data from the 
other. Specifically, the anodic chronoamperometry data is used to obtain the absolute scattering cross-section from the SR-SAXS data, while the SR-SAXS data is used to obtain the specific differential capacitance of the $\mathrm{Pb}$ particles from the electrochemical impedance spectroscopy (EIS) data. Use of the SR-SAXS is necessary, in this study, because additional cathodic reactions occur, which prevent the use of the cathodic faradaic charge passed at each cathodic potential to calculate the deposit mass. Furthermore, the combined SR-SAXS/EIS approach could resolve any morphological dependence on the differential capacitance. However, this is not observed here.

\subsection{Pb Electrodeposition}

Lead electrodeposition has been used to study the fundamental deposition mechanism in both aqueous[34] and room temperature ionic liquids[35]. In the field of quantum dot synthesis[36], PbS particles have been studied and assembled using DES and using solution synthesis methods[18]. While $\mathrm{PbS}$ thin films have been co-deposited using thiourea[37] (a possible DES component), quantum dots have not. Therefore, the aim of this study is to understand $\mathrm{Pb}$ nanoparticle electrodeposition in a $\mathrm{DES}$ towards $\mathrm{PbS}$ quantum dot synthesis. From an fundamental perspective, $\mathrm{Pb}$ was used to: maximize the $\mathrm{X}$-ray contrast in the deposit, to observe any aggregation/coalescence[11] and capacitance change of deposited particles, and to observe how the particle deposit dissolves. Therefore, the in-situ evolutions of the deposit morphology (using SR-SAXS) and of its capacitance (using EIS) are characterized in the potential range: $0.2 \mathrm{~V}$ to $-1.2 \mathrm{~V}$. Since SR-SAXS analysis requires some modifications to a typical small angle scattering (SAS) analysis, a detailed discussion is provided in the Theory section.

\section{Experimental}

\subsection{Materials}

The DES solution was prepared by mixing recrystallized choline Cl- (Sigma-Aldrich) and urea (Sigma-Aldrich) in a 1:2 molar ratio in absolute ethanol. The mixture was left to melt on a hot plate at $90^{\circ} \mathrm{C}$ and under an Ar blanket. Once a homogenous liquid was observed, 0.002 wt.\% $\mathrm{PbCl}_{2}(\sim 0.01 \mathrm{mM})$ was added, mixed and stirred under an $\mathrm{Ar}$ blanket. The $\mathrm{PbCl}_{2} / \mathrm{DES}$ mixture was allowed to dissolve under these conditions for 6 hours to obtain the final, clear solution. This final solution was transferred to a sealed glass container and stored. The 
working and counter electrodes used in these experiments were $180 \mu \mathrm{m}$ thick glassy carbon (HTW) and Pt mesh (Sigma Aldrich), respectively. A leakless $\mathrm{Ag} / \mathrm{AgCl}$ reference electrode (eDaq) was used in the electrochemical cell. Therefore, all voltages reported here are versus $\mathrm{Ag} / \mathrm{AgCl}$.

\section{$2.2 X$-ray Scattering}

Sample rotated small angle X-ray scattering (SR-SAXS) experiments were performed using the custom designed electrochemical cell reported previously and at sample angles of $45^{\circ}, 30^{\circ}$ and $20^{\circ}$.[25] These experiments were performed at the USAXS beamline at sector 9-ID, Advanced Photon Source at an X-ray energy of $18 \mathrm{keV.[38]} \mathrm{These} \mathrm{data} \mathrm{are} \mathrm{on} \mathrm{an} \mathrm{absolute}$ scale when normalized by the sample thickness. Some attenuation was necessary to avoid beam damage in these experiments and was observed by either bubble formation or X-ray induced changes in the open circuit potential. For these experiments, transmissions of $10 \%$ were sufficient to avoid this beam damage. All of the SR-SAXS fitting was performed using the lmfit module for python[39], while the normal SAXS model fitting was accomplished using the Irena package for Igor pro[40].

\subsection{Electrochemistry}

To characterize the Pb-DES system, we report first the cyclic voltammogram of the cell, which was obtained using a scan rate of $50 \mathrm{mV} \mathrm{s}^{-1}$ between $0.2 \mathrm{~V}$ and $-1.3 \mathrm{~V}$, as this is the electrochemical window for the DES at room temperature and without a metal salt.[20] These data are used to qualitatively characterize the electrochemical system. The main focus of this paper is the potential sweep procedure, which employed: chronoamperometry, electrochemical impedance spectroscopy (EIS) and SR-SAXS. In this procedure, the cell was polarized between $0.2 \mathrm{~V}$ and $-1.2 \mathrm{~V}$ at $0.1 \mathrm{~V}$ to $0.2 \mathrm{~V}$ increments. In regions where $\mathrm{Pb}$ is present on the surface, potential steps of $0.1 \mathrm{~V}$ were used, which corresponds to the potential range: $-0.8 \mathrm{~V}$ to $-1.2 \mathrm{~V}$ and back to $-0.6 \mathrm{~V}$. A step of $0.1 \mathrm{~V}$ was chosen because it was small enough to capture significant changes in the EIS and large enough to make the best use of beam time. Steps of either $0.1 \mathrm{~V}$ or $0.2 \mathrm{~V}$ were used outside this range for time constraints, as the EIS did not change significantly. The SR-SAXS/EIS procedure, at each step, is explained in the following section (2.4). Electrodeposition experiments in the main article were performed only after complete dissolution of the substrate. In each case, the potential was 
held at the electrodeposition potential during data collection. Short electrodeposition pulses were not possible and discussed in the Supplementary Section A.2.2.

All of the electrochemical measurements were obtained using a Gamry Reference-600 potentiostat. At each potential, $\Phi$, the cell capacitance was obtained from the potentiostatic electrochemical impedance spectroscopy (EIS) using the Gamry frameworks software. These impedance measurements applied a single sinusoidal single of $10 \mathrm{mV}$ (root-mean-squared) above the DC offset at each potential, $\Phi$, and at a range of frequencies. The frequency range between $0.1 \mathrm{~Hz}<f<10000 \mathrm{~Hz}$ was evaluated, with 10 log-spaced frequencies per decade.

\subsection{SR-SAXS/EIS Procedure}

Key to this approach is the electrochemical and X-ray scattering data collection at each potential step where the cell was polarized to a specified potential, $\Phi$ (in V vs. $\mathrm{Ag} / \mathrm{AgCl}$ ). The time sequence of the EIS and SR-SAXS are shown for one potential (-0.9 V) in Figure 1. At each step, the cell was polarized for $10 \mathrm{~s}$ to allow the system to reach a steady state current. Ideally, the EIS and SR-SAXS should be collected simultaneously. However, rotation of the cell while collecting EIS data can result in unwanted noise. Therefore, the SR-SAXS data collection was started in the middle of the EIS such that the first $45^{\circ}$ SAXS data was finished at the same time. Following the EIS, the cell was held at the potential, $\Phi$, for 17 minutes to allow the rest of the SR-SAXS to finish. Typically, the SR-SAXS was finished $\sim 10$ minutes to 13 minutes before 17 minute polarization completed; this extra time allowed for some growth between SR-SAXS collection, as well as any necessary (USAXS) instrument tuning. Once the 17 minute polarization completed, the cell was polarized to the next $\Phi$ value and the procedure repeated. Therefore, the procedure can be summarized as a chronological sequence of: chronoamperometry, EIS, chronoamperometry, with some dead time in-between steps.

\section{Theory}

\subsection{SR-SAXS}

In a typical small angle scattering experiment, the scattered intensity is measured as a function of the scattering vector, $\mathbf{q}$. The shape of $I(\mathbf{q})$ is then used to obtain the size and shape of the scattering phases. In the case where the scattering phases are anisotropic and confined to a surface, a grazing transmission geometry has been shown to be a powerful approach to resolving their size and shape by collecting X-ray scattering data at different sample 
orientations.[25] Here, the sample angles were chosen to maximize the scattering signal and small angle scattering was collected at lower $q$ to resolve larger phases. In all of the SR-SAXS data, the absolute intensity was obtained by initially assuming a sample thickness, $T_{s}$, of $1 \mathrm{~mm}$; this is a necessary correction in the data reduction software. As the $\mathrm{Pb}$ deposit is confined to the surface, the mean height of the particles, $\bar{H}$, is obtained from the model fitting and used to obtain the absolute scattering cross-section[41]. During nucleation, $\bar{H}$ could not be extracted from a model fit and therefore the intensity units are arbitrary (a.u.). The absolute intensity of any small angle scattering data scales with the square of the total volume and contrast of the scattering population.[42] This means that without knowledge of one, it is difficult to know the quantity of the other (within a useful margin). For an electrochemist, a convenient solution to this problem is to combine the electrochemical data with the small angle scattering (SAS) data. This procedure is unfortunately not common and therefore deserves some introduction.

By combining the faradaic charge from the chronoamperomentry data with the density of the deposit, one could calculate the total volume. The only requirements are that the faradaic reactions are known and quantifiable and the density of the deposit is known. In this case, the deposit volume can be taken directly from the anodic current and put into the SAS model. However, in this study and in many real systems, there are side reactions $\left(\mathrm{O}_{2}\right.$ reduction $)$ that prevent the faradaic cathodic charge from being used to calculate the total volume. The $\mathrm{O}_{2}$ reduction is irreversible in the potential window evaluated here and will be discussed in Section 4.1. Therefore, the anodic charge contains only $\mathrm{Pb}$ dissolution at $-0.6 \mathrm{~V}$ and is used to calculate the total particle volume. With the total particle volume and scattering length density known, the scattering length density of the surrounding solution can be calculated and compared with expected theoretical values. Furthermore, if one can assume this stays approximately constant, the volume (and therefore mass) of the particles at each potential can be calculated and used with EIS measurements to obtain the specific differential capacitance. In this manner, the chronoamperometry data is used to extract more information from the SR-SAXS, which can then be combined with EIS data.

\subsubsection{Nucleation/Growth/Aggregation (-0.8 V)}

On the initial cathodic sweep, SAXS was first observed at $-0.8 \mathrm{~V}$. Unlike subsequent data, the 
morphology of the $\mathrm{Pb}$ deposit evolves over time and cannot be fit to a single model. For this reason, no shape determination of the deposit morphology at $-0.8 \mathrm{~V}$ can be made and the scattering data are referred to as SAXS data, as each curve was fit separately. For this reason, only the evolution in particle structure can be extracted during this time and nothing conclusive can be reported about the shape of the particles. In these data, the slit-smeared SAXS could have some anisotropy but cannot be resolved. Therefore, the model smearing was performed by assuming particle isotropic scattering. Consequently, only the radius of gyration, $R_{g}$, of the particles and any hierarchal structure can be reliably reported. For aggregated particles, their structure is related to their fractal dimension, $d_{m}$.[43] The unified approximation is ideal for modeling such systems since it combines the robust Guinier and Porod relationships with the power-law scattering one expects from a fractal-like aggregates.[44, 45]

After 2 minutes, the first SAXS data $\left(45^{\circ}\right)$, revealed only very weak scattering that cannot be reliably modeled. The following scan $\left(30^{\circ}\right)$, collected 4 minutes after, revealed a clear Guinier knee and was modeled using a single-level unified equation:

1.

$$
I_{p}(q)=G_{p} e^{\frac{-q^{2} R_{g}{ }^{2}}{3}}+B_{p}\left[\frac{\left(\operatorname{erf}\left(\frac{q R_{g}}{\sqrt{6}}\right)\right)^{3}}{q}\right]^{4}+b c k g
$$

2.

$$
B_{p}=\frac{2 \pi G_{s} A_{p}}{V_{p}^{2}}
$$

where $G_{p}$ is proportional to the volume squared and contrast squared of the primary particles, $R_{g}$ is the radius of gyration of the primary particles, $A_{p}$ and $V_{p}$ are the surface area and volume of spheres having a radius equal to $R_{g} \sqrt{5 / 3}$ and $b c k g$ is a constant representing the background scattering. To ensure the feasibility of the model, $B_{p}$ is calculated from $G_{p}$ (Equation 2) and assumes the particles have a surface to volume ratio of a sphere. From the model fit of Equation 1, an $R_{g}$ of $58 \mathrm{~nm} \pm 3 \mathrm{~nm}$ was found for the SAXS data obtain 4 minutes after the nucleation. 
Six minutes after nucleation, the SAXS data revealed a power-law decay in intensity that preceded the Guinier knee. Therefore, the data were fit the two-level unified equation:

3.

$$
I_{A}(q)=B_{A} e^{\left(\frac{-q^{2} R_{g}^{2}}{3}\right)}[q]^{d_{m}}+I_{p}(q)
$$

where $d_{m}$ is the mass fractal dimension and $B_{A}$ is a scaling constant. From the model fitting of Equation 3 to the SAXS data an $R_{g}$ of $62 \mathrm{~nm} \pm 4 \mathrm{~nm}$ and $d_{m}$ of $2.5 \pm 0.5$ were found. The difference between Equations 1 and 3 is the lack of particle structure in the former (4 minutes). Therefore, from the fits of Equations 1 and 3 the size and structure of the particle deposit before and after aggregation are obtained, respectively.

\subsubsection{Particle Growth}

Unlike ordinary slit-smeared SAXS, the slit-smeared SR-SAXS can resolve surface anisotropy. In the potential range: $-0.9 \mathrm{~V}$ to $-1.2 \mathrm{~V}$ and $-1.2 \mathrm{~V}$ to $-0.7 \mathrm{~V}$, all three of the SR-SAXS data $\left(45^{\circ}, 30^{\circ}\right.$ and $\left.20^{\circ}\right)$, collected at each potential, are fit to a system of oriented ellipsoids that are polydisperse in size. From each model fit, the time-averaged physical dimensions and volume fraction of the $\mathrm{Pb}$ deposit over the course of 6 minutes are obtained. Therefore, this analysis is limited to systems with nucleation and growth kinetics that are sufficiently slow. In cases where sufficient particle nucleation is present, one can expect to see a broadening of the size distribution as smaller particles are deposited. On the other hand, if significant growth occurs over the scan period, the faradaic current should be incorporated in the model fit to obtain the particle size and shape accurately. Such a formulation is discussed later in Section 4.2.2.a where particle growth is included in the model fitting. In the potential range: $-0.9 \mathrm{~V}$ to $-1.2 \mathrm{~V}$ and $-1.2 \mathrm{~V}$ to $-0.7 \mathrm{~V}$, both nucleation and growth are minor effects in these experiments. However, it is important for an experimenter to consider these effects and therefore they will be discussed further in Section 4.

The scattered intensity from an oriented spheroid, is the same as that of a perfect sphere with an effective radius, $R_{\text {eff: }}$ [42] The value of $R_{\text {eff }}$ is calculated using the scattering vector, $\mathbf{q}_{R}$, radius, $R$, and aspect ratio, $A R$ by the equation:

4.

$$
R_{e f f}\left(\mathbf{q}_{R}, R\right)=\sqrt{R^{2}\left(q_{x} /\left|\mathbf{q}_{R}\right|\right)^{2}+R^{2}\left(q_{y} /\left|\mathbf{q}_{R}\right|\right)^{2}+R^{2} A R^{2}\left(q_{z} /\left|\mathbf{q}_{R}\right|\right)^{2}}
$$

where the $x, y, z$ directions and $\mathbf{q}$ vectors are shown in Figure 1 , where the $\mathbf{q}$ vectors are 
oriented rather than the sample for simplicity.[42] For the slit-smeared geometry used in these experiments, the scattered intensity, $I$, was collected as a function of the scattering angle perpendicular to the surface, $\theta_{\perp}$. The intensity, $I$, at each $\theta_{\perp}$ was slit-smeared with the scattered intensity parallel to the glassy carbon surface, which is a function of the parallel scattering angle, $\theta_{\|}$. With the sample angle, $\alpha$, as well as $\theta_{\perp}$ and $\theta_{\|}$, obtained from the instrument geometry, the scattering vector $\mathbf{q}_{\mathbf{R}}$ is calculated by the Equations:

5.

6.

7.

8.

$$
\begin{array}{r}
\mathbf{q}_{R}\left(q_{\|}, q_{\perp}, \alpha\right)=q_{x} i+q_{y} j+q_{z} k \\
q_{x}=\frac{2 \pi\left(\cos \left(-\alpha-\theta_{\perp}\right) \cos \theta_{\|}-\cos \alpha\right)}{\lambda} \\
q_{y}=\frac{2 \pi\left(\sin \theta_{\|}\right)}{\lambda} \\
q_{z}=\frac{2 \pi\left(\sin \left(-\alpha-\theta_{\perp}\right) \cos \theta_{\|}-\sin \alpha\right)}{\lambda}
\end{array}
$$

With the values of $\mathbf{q}_{\mathbf{R}}$ and the slit-length, $q_{\text {slit }}$, known, the scattered intensity from a size distribution of particles can be calculated. The slit-smeared scattered intensity from a Gaussian distribution, $P(R)$, of oriented ellipsoid is then:

9.

$$
I_{s m r}\left(q_{\perp}, \alpha\right)=\frac{K}{\sin \alpha} \int_{0}^{q_{s l i t}} I\left(\mathbf{q}_{R}\left(q_{\|}, q_{\perp}, \alpha\right)\right) d q_{\|}
$$

10.

$$
I\left(\mathbf{q}_{R}\left(q_{\|}, q_{\perp}, \alpha\right)\right)=\int_{0.01 R}^{100 R} V_{p} F^{2}\left(\mathbf{q}_{R}, R\right) P(R) d R
$$

11.

$$
F\left(\mathbf{q}_{R}, R\right)=\left[\frac{3\left[\sin \left(\left|\mathbf{q}_{R}\right| R_{e f f}\left(\mathbf{q}_{R}, R\right)\right)-\left|\mathbf{q}_{R}\right| R_{e f f}\left(\mathbf{q}_{R}, R\right) \cos \left|\mathbf{q}_{R}\right| R_{e f f}\left(\mathbf{q}_{R}, R\right)\right]}{\left[\left|\mathbf{q}_{R}\right| R_{e f f}\left(\mathbf{q}_{R}, R\right)\right]^{3}}\right]
$$

where the subscripts $\|$ and $\perp$ are directions of the scattering vector, parrallel to the surface and perpendicular to the surface, respectively, $\alpha$ is the sample angle, $q$ and $\mathbf{q}$ are the reciprocal space magnitude and vector, respectively, $K$ is a scaling constant $\left(\mathrm{cm}^{-4}\right), R$ is the radius of the spheroid, $A R$ is the aspect ratio, $V_{p}$ is its volume and $P(R)$ is a gaussian function that scales the scattering from particles of size $R$, using the standard deviation, $\sigma$, and mean particle radius, $\bar{R} .[42,46]$; the units of $P(R)$ are $\mathrm{nm}^{-1}$. The scaling constant, $K$, is proportional to the total particle volume per irradiated volume (i.e. volume fraction), $v$, and X-ray contrast, $\Delta \rho$. These values can be extracted from $K$ when one is known. From the model fit of Equation 9 to the SR-SAXS data, the values of: $K, A R, \bar{R}$ and $\sigma$ are extracted 
as a function of potential. It is important to note that Equation 9 is two dimensional in reciprocal space. This allows the scattered intensity obtained at different sample angles, $\alpha$, to be fit to a single equation.

\subsection{Electrochemical Impedance Spectroscopy (EIS)}

The EIS data were fit to an equivalent circuit that contains the solution resistance, $R_{s}$, in series with a parallel circuit that represents the surface charge transfer resistance, $R_{t}$, and capacitance, which is taken to be a constant phase element, CPE. The equivalent circuit was fit to these EIS data in the frequency range of $0.1 \mathrm{~Hz}<f<10000 \mathrm{~Hz}$ and given by the equation:

$$
Z(\omega)=R_{S}+\left(1 / R_{t}+(\omega \sqrt{-1})^{\gamma} Q\right)^{-1}
$$

where $\omega$ is the angular frequency, $Q$ has units of $s^{\gamma} \Omega^{-1}$ and $\gamma$ is an exponent that approaches one for a narrow distribution of resistances, $R_{t}$.[47] In Equation 12, the value of $R_{t}$ represents the charge transfer resistance at the surface, while $Q$ is related to the total surface charge. In Equation 12, a constant phase element (CPE) is used instead of a capacitor to account for a

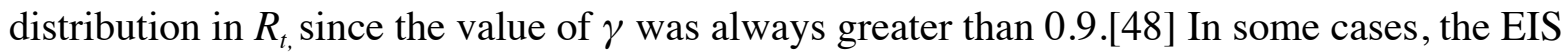
model fits could be improved by accounting electroactive adsorption, as reported previously.[25] These EIS data were obtained immediately following nucleation and growth $(-0.9 \mathrm{~V})$ and the onset of dissolution $(-0.7 \mathrm{~V})$. However, only minor improvements to the model fit were obtained with relatively large errors associated with the fit parameters. These model fits can be found in the Supplementary Section A.1.

\section{Results and Discussion}

\subsection{Cyclic Voltammetry}

The Pb-DES system was characterized by three sequential cyclic voltammograms shown in Figure 1. There are three notable observations: 1) there is one cathodic peak and one anodic peak, 2) there is more cathodic charged passed for each cycle and 3) there is an increase in the peak current with scan number. As the current, $I_{C}$, was swept to cathodic potentials less than $-0.8 \mathrm{~V}$, the $\mathrm{Pb}$ precursor was reduced on the glassy carbon surface. The deposited $\mathrm{Pb}$ particles continue to grow on the anodic sweep until $\sim-0.7 \mathrm{~V}$, at which point the $\mathrm{Pb}$ deposit oxidizes and is removed from the surface; this is confirmed with SAXS measurements. 
Therefore, the $\mathrm{Pb}$-DES system is reversible, which makes it an ideal system to study nucleation, growth and dissolution in-situ without changing the sample cell.

In all scans, there is slightly more cathodic charge passed than anodic charge. In the first scan there is $13 \%$ more charge passed, while only $6 \%$ more cathodic charge is passed in the final scan. This extra cathodic charge is attributed to the presence of small amounts of oxygen[49] in the solution that was unavoidable in these experiments. The presence of $\mathrm{O}_{2}$ has two major consequences for the $\mathrm{Pb}$-DES system: 1) it precludes precise deposit mass determination from the cathodic region of Figure 2, as $\mathrm{O}_{2}$ is reduced[49] at the same time as the $\mathrm{Pb}$ precursor and 2) a galvanic couple can form between deposited $\mathrm{Pb}$ and $\mathrm{O}_{2}$, which will dissolve the $\mathrm{Pb}$ if the cell is not polarized; this is demonstrated and discussed further in the Supplementary Section A.2. Based on previous work in a 1:2 choline $\mathrm{Cl}^{-}$:ethaline DES, an $\mathrm{O}_{2}$ reduction peak in the $\mathrm{CV}$ was observed between $-0.63 \mathrm{~V}$ and $-0.78 \mathrm{~V}$ vs. $\mathrm{Ag} / \mathrm{AgCl}[49]$, which is very near that of $\mathrm{Pb}$ oxidation. It was estimated that during the polarization sweep, the $\mathrm{Pb}$ current efficiency, $\eta$, was $\sim 70 \%$ in the cathodic region. This approximation is detailed in the Supplementary Section A.3 and is used to estimate particle growth during the SR-SAXS data set.

The increase in peak current could be attributed to an increase in the diffusion coefficient of the $\mathrm{Pb}$ precursor near the glassy carbon, though this was not explored further[50]. However, it will be shown that there is a significant increase in the concentration of the $\mathrm{Pb}$ precursor, near the glassy carbon surface, following electrodeposition, which is expected to reduce ion mobility[50].

\subsection{SR-SAXS and EIS Results}

\subsubsection{SAXS}

The model fits shown in Figure 3 are unable to extract any shape information from the particle deposit. Based on the SR-SAXS modeling and the fact that these particles are confined to the surface, the particle aggregate could be slightly anisotropic but is difficult to determine from only one SAXS data set. These aggregates coalesce to form individual phases, which are modeled as oriented ellipsoids at subsequent potentials.

\subsubsection{SR-SAXS}

The oriented spheroid model fits are shown in Figure 4 and the evolution of the values of $\overline{\boldsymbol{R}}$, 
$\sigma$ and $A R$ during the potential sweep are shown in Figure 5. From the SR-SAXS model fitting, the aspect ratio showed no clear trend with potential and had an average value of $0.75 \pm 0.03$. The standard deviation of the size distribution, $\sigma$, increases between $-0.9 \mathrm{~V}$ and $-1.0 \mathrm{~V}$ on the initial cathodic scan. This increase is only slightly outside of the fit error and could be associated with additional particle nucleation between $-0.9 \mathrm{~V}$ and $-1.0 \mathrm{~V}$. Beyond $-1.0 \mathrm{~V}$ and into the anodic scan, $\sigma$ shows very little trend outside of the fit error. Therefore, the width of the particle size distribution does not change within the fit error beyond $-1.0 \mathrm{~V}$ on the cathodic scan. Keeping in mind that the scattered intensity of a particle scales with the square of its volume[42], it is difficult to resolve smaller particles that nucleate in the presence of larger ones. Therefore, any additional small particles could not be detected beyond $-1.0 \mathrm{~V}$. The aspect ratio (and therefore the shape of the particles) remains in the range: $0.75 \pm 0.05$, including the fit error shown in Figure 5. The mean radius of the $\mathrm{Pb}$ deposit increased from $110 \mathrm{~nm} \pm 2 \mathrm{~nm}$ to $132 \mathrm{~nm} \pm 1 \mathrm{~nm}$ (Figure 5) before the particles began to dissolve at $-0.7 \mathrm{~V}$. Therefore, as time passes during the potential sweep, the particle growth is considered to be the primary change in particle morphology, with little change in particle shape and number outside of the fit error.

Overall, the mean particle radius increases by $\sim 20 \mathrm{~nm}$ within the potential sweep, which was evaluated at 9 different potentials with SR-SAXS. Referring to Figure 1, 40\% (by time) of this growth occurs during SR-SAXS collection, while the remaining $60 \%$ occur after each SR-SAXS collection sequence. Therefore, there is some growth smearing within each SR-SAXS data set, which is not accounted for in Equation 9 and is discussed in the following sub-section. Because of the relatively slow growth kinetics, the growth-smearing is most pronounced just after nucleation $(-0.9 \mathrm{~V})$.

\subsection{2.a Growth Smearing:}

Growth smearing consists of changes in particle size that occurs during all three scans. It is important to note that this phenomena is not specific to SR-SAXS. Rather, it is present for all in-situ SAXS measurements where particles are actively growing. The consequence of this effect depends on the growth kinetics and the time of measurement. Here, the particle growth is fastest immediately following nucleation (Figure 5). Therefore, the particle growth is accounted for in the SR-SAXS data collected at $-0.9 \mathrm{~V},-1.0 \mathrm{~V}$ and $-1.1 \mathrm{~V}$ during the cathodic 
scan and compared with Equation 9 to demonstrate this effect. Beyond these potentials, the change in $\bar{R}$ is much less and therefore not evaluated. The difference in the fit parameters with and without including particle growth are greatest at $-0.9 \mathrm{~V}$ and decrease until the differences are very close to the fit error at $-1.1 \mathrm{~V}$.

The total cathodic charge measured during each scan can be used estimate the particle growth. This faradaic charge changes four of the model parameters: the volume fraction, $v$, aspect ratio, $A R$, mean radius, $\bar{R}$ and width of the size distribution, $\sigma$. Moreover, it is assumed that the particles grow equally in all directions since the value of $A R$ does not change significantly during the potential sweep (Figure 5). The formulation is such that all modifications to the volume fraction, $v, \bar{R}$ and $\bar{H}$ are made to the second and third SAXS profiles obtained at $30^{\circ}$ and $20^{\circ}$. Therefore, all other fit parameters are associated with the first SAXS profile $\left(45^{\circ}\right)$ and the values of $v, \bar{R}$ and $\bar{H}$ are modified for the subsequent scans used in the model fitting.

From the chronoamperograms, the total current density passed is converted to $\mathrm{Pb}$ volume to give the specific faradaic volume added in the second, $\hat{v}_{F_{30}}$, and third scans, $\hat{v}_{F_{20}}$, by the equations:

13.

$$
\hat{v}_{F_{30}}=\eta \frac{M}{n F d} \int_{t_{0}}^{t_{45}} i(t) d t
$$

14.

$$
\hat{v}_{F_{20}}=\eta \frac{M}{n F d} \int_{t_{0}}^{t_{30}} i(t) d t
$$

where $\eta$ is the faradaic current efficiency $(\eta=0.7)$ for $\mathrm{Pb}$ reduction, $M$ is the molecular weight of $\mathrm{Pb}, n$ is $2, F$ is Faraday's constant, $d$ is the density $\left(\mathrm{g} \mathrm{cm}^{-3}\right), i(\mathrm{t})$ is the measured current density and $t_{0}$ is the start time of the SR-SAXS set (or first scan), $t_{45}$ is the end time of the first scan $\left(45^{\circ}\right)$ and $t_{30}$ is the end time of the second scan $\left(30^{\circ}\right)$. The units of $\hat{v}_{F}$ are $\mathrm{cm}$, until it is divided by the mean height of the $\mathrm{Pb}$ particles, $\bar{H}$, at which time it is the volume fraction added at the sample angle, $\alpha, v_{F_{\alpha}}=\frac{\hat{v}_{F_{\alpha}}}{\bar{H}_{\alpha}}$. If we also assume uniform particle growth, the mean radius, $\bar{R}$, must also increase by $\Delta \bar{R}$, which can be calculated from the equations:

$$
\Delta \bar{R}_{30}=\left[\bar{R}^{3}\left[1+\frac{v_{F_{30}}}{v_{45}}\right]\right]^{1 / 3}-\bar{R}
$$


16.

$$
\Delta \bar{R}_{20}=\left[\bar{R}^{3}\left[1+\frac{v_{F_{20}}}{v_{45}}\right]\right]^{1 / 3}-\bar{R}
$$

where $v_{45}$ is a fit parameter and represents the volume fraction at the end of the first scan. Finally, the volume fraction of the second, $v_{30}$, and third, $v_{20}$, scans are modified by the equations;

17.

$$
\begin{aligned}
& v_{30}=v_{45}+v_{F_{30}} \\
& v_{20}=v_{45}+v_{F_{20}}
\end{aligned}
$$

18.

To evaluate the difference between models with and without growth presented here, the SR-SAXS data at $-0.9 \mathrm{~V}$ and $-1.0 \mathrm{~V}$ were fit to the equation:

19.

$$
\begin{array}{r}
I_{s m r}\left(q_{\perp}, \alpha\right)=\frac{K_{\alpha}}{\sin \alpha} \int_{0}^{q_{s l i t}} I\left(\mathbf{q}_{R}\left(q_{\|}, q_{\perp}, \alpha\right)\right) d q_{\|} \\
K_{\alpha}=\frac{v_{\alpha}\left[1-v_{\alpha}\right] \Delta \varrho^{2} \bar{H}}{T_{s}}
\end{array}
$$

20.

where $\Delta \varrho^{2}$ was taken to be that of $\mathrm{Pb}$ in Equation 23. Differences in the parameters, $v, \bar{R}, \sigma$ and $A R$ between Equation 9 and Equation 19 are tabulated in Table 1. It is important to note the scaling correction in Equaton 20 that includes $T_{s}$ and $\bar{H}$, which are used to put $I_{s m r}\left(q_{\perp}, \alpha\right)$ on an absolute scale. The aspect ratio, $A R$, is consistently higher than the $A R$ obtained in the original model fit (Figure 5) until $-1.1 \mathrm{~V}$ where AR is within the range: 0.75 \pm 0.05 . On the other hand, the values of $\bar{R}, \sigma$ and $v$ are consistently lower than those obtained without including particle growth, until $-1.1 \mathrm{~V}$ where the differences are very near the combined fit error. Therefore, the overall effect of growth-smearing is to artificially reduce the aspect ratio and increase the particle size distribution and volume when particle growth is not included in the model fit. The differences in model fit can be seen in Figure 6 and decrease with the order: $-0.9 \mathrm{~V},-1.0 \mathrm{~V}$ and $-1.1 \mathrm{~V}$. In general, lower chi-square values were obtained by including the faradaic growth for the $\mathrm{Pb}$-DES system.

\subsection{2.b Fit Deviations}

The fit of Equation 9 deviates from data at low- $q\left(q<0.001 \mathrm{~A}^{-1}\right)$ where the model fit is either within or near the measurement error. The source of these deviations is worth some discussion, as they highlight some limitations to SR-SAXS. In the previous section, growth 
smearing was discussed and arises due to the relatively long scan times. The model fit represented by Equation 9 also does not account for: particle structure, near-interface solution phases or small surface features. These are aspects of the system which could improve the model fit but are too ambiguous to model with enough certainty.

At very low- $q\left(q<0.001 \mathrm{~A}^{-1}\right)$, the measured intensity $\left(45^{\circ}\right.$ and $\left.30^{\circ}\right)$ increases with $q$ and can be attributed to particle correlations, which influence the scattered intensity at volume fractions greater than $\sim 0.06[42]$. However, the absence of a clear peak in intensity, suggests that there is very little order to the deposited particles. Near-interphase phases that were observed in a different DES[25] could not be accounted for in this study. In the previous study, the SR-SAXS from multilayer perturbations could be modeled using a monte-carlo approach. With today's computers, this approach is too computationally expensive for slit-smeared SAXS that spans several decades in $q$. These perturbations are very anisotropic and might explain the very different small angle scattering at low- $q$. Finally, it is impossible to differentiate between surface features and isolated particles in the size distribution since the number of particles per aggregate is low. With these considerations in mind, the proposed model is sufficient to extract the change in volume, shape and particle dimensions in the cathodic region of Figure 2.

\subsection{2.c Dissolution}

From the cyclic voltammogram in Figure 2, one can expect that the $\mathrm{Pb}$ phases will dissolve at $-0.6 \mathrm{~V}$. Indeed, this is observed in the SR-SAXS. At $-0.6 \mathrm{~V}$, the general shape of the scattered intensity remains the same as before but is significantly lower in scale compared with $-0.7 \mathrm{~V}$. This rapid decrease in intensity prevents any reliable modeling of the data. Because the shape of the scattered intensity is constant during dissolution, we conclude that the particle aggregates dissolve intact at $-0.6 \mathrm{~V}$. At subsequent anodic potentials, no scattering signal, above the background, could be observed.

\subsubsection{Electrochemical Impedance Spectroscopy (EIS)}

All of the EIS data can be fit to the same equivalent circuit to extract the total surface capacitance and charge transfer resistance at each potential. The model fit of Equation 12 to select EIS data is shown in Figure 7a (cathodic sweep) and Figure 7b (anodic sweep). From each model fit, an average solution resistance, $R_{s}$, of $152 \pm 14 \Omega$ was observed, while the 
charge transfer resistance decreased by an order of magnitude in the presence of the $\mathrm{Pb}$ particles. The fit parameters in Equation 12 are used to calculate the overall surface capacitance, $C$.

For faradaic systems, $C$, can be calculated from the fit parameters of Equation 12 by the equation:

$$
\mathrm{C}=\left[Q\left[\frac{1}{R_{t}}+\frac{1}{R_{s}}\right]^{\gamma-1}\right]^{1 / \gamma}
$$

where all parameters are taken from the model fit of Equations 12.[48] All values of $C$ were initially normalized by the area of the working electrode, $A_{W E}$, to obtain the value of $C_{W E}$, which is shown in Figure 8. In the range: $0.2>\Phi>-0.5$, the value of $C_{W E}$ is approximately constant at $8.3 \mu \mathrm{F} \mathrm{cm}^{-2}$ on the cathodic sweep and $8.8 \mu \mathrm{F} \mathrm{cm}^{-2}$ on the anodic sweep. These values are within $\pm 2 \mu \mathrm{F} \mathrm{cm}^{-2}$ of those obtained from the same DES with $\mathrm{Hg}$ electrode[28] and a diol-containing DES onto glassy carbon[27]. In both these studies, the differential capacitance behavior $[27,28]$ in the cathodic regime did not change significantly and therefore the capacitance of the glass carbon, $\hat{C}_{g c}$, is considered to be $8.5 \mu \mathrm{F} \mathrm{cm}$ throughout the potential region evaluated here. This assumption allows for the removal of the glassy carbon capacitive contribution to $C_{W E}$.

\subsection{Particle Aggregation}

The presence of $\mathrm{Pb}$ nanoparticles are observed in the SAXS data and shown in Figure 3. In the first 4 minutes, isolated $\mathrm{Pb}$ phases are observed without any hierarchal structure. These phases have an $R_{g}$ of $60 \mathrm{~nm}$, which is a function of both their size and shape[42]. As the particle shape cannot be determined during this time, it is difficult to relate $R_{g}$ to a physical dimension.

If, for example, the particles are approximately spherical, they would have a radius of $77 \mathrm{~nm}$. In the following scan (6 minutes), these particles have a fractal-like hierarchal structure, which is characterized of a mass-fractal aggregate.[43] The size of these aggregates could not be resolved with certainty but are expected to be greater than $1 \mu \mathrm{m}$, based on the minimum $q$ values evaluated. Based on the volume distribution of the subsequent coalesced particles, a minimum of 1 to 8 particles are expected in each coalesced phase. This relatively low number 
density of particles is expected for deposition at low overpotentials [51].

The relatively slow aggregation observed at $-0.8 \mathrm{~V}$ after electrodeposition was also observed previously with Ag.[25] In the case of Ag, agglomerate scattering dissipated after 3 minutes and the agglomerates were much more compact after 6 minutes with no clear mass fractal scattering. Therefore, the mobility and aggregation of $\mathrm{Pb}$ is slower than with $\mathrm{Ag}$ at room temperature. Considering the Stokes-Einstein relationship for particle mobility, the higher viscosity in choline:urea DES compared with choline:ethylene glycol[15], as well as larger $\mathrm{Pb}$ primary particle sizes both contribute to slower particle transport and aggregation[11]. Once agglomerated, the particles form hard-aggregates and coalesce into a solid phase to minimize the overall surface area[11,52]. Therefore total coalescence is observed is observed with $\mathrm{Pb}$, rather than partial coalescence.[25]

\subsection{Total Volume Fraction Calculation}

By combining the electrochemical data and SR-SAXS, it is possible to calculate the overall volume fraction, $v_{P b}$, of deposited particles and X-ray contrast, $\Delta \rho$. Though this calculation requires some assumptions about the composition of the deposit and the faradaic current, it is useful to obtain reasonable estimates. In order to estimate $v_{P b}$, the particle deposit is assumed to be pure $\mathrm{Pb}$, though some lead-oxide is expected.[35] In addition, it is assumed that the total current passed at $-0.6 \mathrm{~V}$ (anodic cycle) represents the faradaic dissolution of $\mathrm{Pb}$ or $\mathrm{PbO}_{2}$. However, from Figure 2, it is known that irreversible cathodic reactions are present, which can cause some dissolution of the $\mathrm{Pb}$ deposit at low overpotentials. Therefore, the total volume of deposited $\mathrm{Pb}, V_{P b}$, is considered a minimum value and is calculated by the Equation:

22.

$$
V_{P b}=M \frac{\int_{0}^{t_{f i n a l}} i(t) d t}{z F d}
$$

where $t_{\text {final }}$ is chosen to be time at which no small angle scattering was observed $(-0.5 \mathrm{~V}), z$ is 2, $M$ is molecular weight of $207.2 \mathrm{~g} \mathrm{~mol}^{-1}(\mathrm{~Pb})$ or $239.2 \mathrm{~g} \mathrm{~mol}^{-1}\left(\mathrm{PbO}_{2}\right)$ and $d$ is the density of $11.34 \mathrm{~g} \mathrm{~cm}^{-3}(\mathrm{~Pb})$ or $9.64 \mathrm{~g} \mathrm{~cm}^{-3}\left(\mathrm{PbO}_{2}\right)$. The electrochemical volume fraction is obtained by multiplying the area of the glassy carbon by the volume-mean height of the particles at $-0.7 \mathrm{~V}$, which is found by SR-SAXS modeling to be $200 \mathrm{~nm}$. With these assumptions, the volume fraction of deposited lead is estimated to be between $12 \%(\mathrm{~Pb})$ and $16 \%\left(\mathrm{PbO}_{2}\right)$ just before 
dissolution. These values indicate that the $\mathrm{Pb}$ phases are the dilute, scattering phase. However, these values are high enough to expect some structuring in the SR-SAXS data, as mentioned in Section 4.2.2.b.

With the value of $v_{P b}$ known, the $\mathrm{X}$-ray contrast, $\Delta \rho$, can be calculated by the equation:

$$
\Delta \rho=\rho_{P b}-\rho_{D E S}=\sqrt{\frac{T_{S} K}{\left(v_{P b}-v_{P b}^{2}\right) \bar{H}}}
$$

where $K$ is the scaling constant and $\bar{H}$ is the volume-mean height of the particles at $-0.7 \mathrm{~V}$. From the calculation of a minimum value for $v_{P b}$, the minimum scattering length density of the surrounding solution, $\varrho_{D E S}$, is estimated to be $16.7 \times 10^{10} \mathrm{~cm}^{-2}\left(\mathrm{PbO}_{2}\right)$ and $18.4 \times 10^{10} \mathrm{~cm}^{-2}$ $(\mathrm{Pb})$. These values are greater than the theoretical scattering length density of the Pb-DES solution, $11.3 \times 10^{10} \mathrm{~cm}^{-2}$. Therefore, immediately following electrodeposition, there is an accumulation of dissolved $\mathrm{Pb}$ ions near the glassy carbon surface, as was observed after $\mathrm{Ag}$ electrodeposition.[25]

If this elevated concentration is assumed to be constant during the polarization sweep, the volume fraction, $v_{P b}$, of the $\mathrm{Pb}$ deposit is calculated from each model fit by the equation:

$$
v_{P b}\left(1-v_{P b}\right)=\frac{T_{S} K}{\bar{H} \Delta \rho^{2}}
$$

where the lowest root of Equation 24 is the volume fraction of $\mathrm{Pb}$ and the deposit is assumed to be elemental $\mathrm{Pb}$. The values of $v_{\mathrm{Pb}}$ are shown in Figure 9. The value of $v_{\mathrm{Pb}}$ increases rapidly following nucleation, as both particle growth and some nucleation occur. During the anodic sweep, the particles continue to grow until $-0.7 \mathrm{~V}$, where some of the deposit begins to dissolve.

\subsection{Specific Capacitance}

In the potential range: $-0.8 \mathrm{~V}$ to $-1.2 \mathrm{~V}$, the values of $C_{W E}, \hat{C}_{G C}$ and $v_{P b}$ can be used to obtain the specific capacitance, $C_{m}$. First, the total capacitance from the $\mathrm{Pb}$ deposit is obtained by removing the capacitive contribution from the glassy carbon. The active area of glassy carbon, in the presence of the $\mathrm{Pb}$ deposit, $A_{G C}$, is obtained by subtracting the cross-sectional area of the $\mathrm{Pb}$ deposit from the total area of the working electrode. With knowledge of the volume fraction of the $\mathrm{Pb}$ deposit, $v_{P B}, A_{G C}$ is obtained by the equation:

25.

$$
A_{G C}=\left(1-3 / 2 v_{P b}\right) A_{W E}
$$


where any cross-sectional area of the $\mathrm{Pb}$ deposit is removed from the total area. Equation 25 assumes that there is no capacitive behaviour between the glassy carbon and $\mathrm{Pb}$ deposit. Therefore, the total capacitance of the $\mathrm{Pb}$ deposit is obtained by the Equaitons:

26.

27.

$$
\begin{gathered}
C_{P b}=C-\hat{C}_{G C} A_{G c} \\
C_{m}=\frac{C_{P b}}{v_{P b} A_{W E} \bar{H} d_{P b}}
\end{gathered}
$$

where the values of elemental $\mathrm{Pb}$ are used, as these values are $10 \%$ higher than for $\mathrm{PbO}_{2}$. The maximum differential capacitance is shown in Figure 10, where $C_{m}$ has a maximum value at $-1.0 \mathrm{~V}$ in the anodic and cathodic scans. However, the normalization reveals that $C_{m}$ is lower in the anodic scan as the specific surface area of the deposit decreases due to coalescence. Because the values of $v_{P b}$ are minimum value estimates, the values of $C_{m}$ are therefore maximum values.

The absolute values of $C_{m}$ are two orders of magnitude lower than typical supercapacitor materials[24], owing to the high density and molecular weight of $\mathrm{Pb}$. The differential capacitance is markedly different than that obtained from a planar $\mathrm{Hg}$ electrode, where the capacitance is constant in the cathodic region[28]. It is clear from Figure 3 and Figure 10 that the particle capacitance at $-0.9 \mathrm{~V}$ is insufficient to achieve electrostatic stability of the $\mathrm{Pb}$ particles. From Figure 10, the specific capacitance at $-1.0 \mathrm{~V}$, during the cathodic sweep, is higher than that obtained on the anodic sweep. Though this difference is within the experimental error, it is worth noting since particle coalescence is also observed and expected to continue between the two measurements. This coalescence is expected to reduce the total available area of the $\mathrm{Pb}$ particles. Therefore, the specific differential capacitance shown in Figure 10 reveals an optimum potential for stable nanoparticle electrodeposition, as well as particle coalescence.

The SAXS data following electrodeposition at $-1.0 \mathrm{~V}$ and $-0.8 \mathrm{~V}$, at $30^{\circ}$ sample angles, are shown in Figure 11. These scattering curves revel a Guinier region that is much smaller than at-1.0 $\mathrm{V}$ than at $-0.8 \mathrm{~V}$. Using the Guinier approximation, the particles obtained at $-1.0 \mathrm{~V}$ have an $R_{g}$ of $36 \mathrm{~nm}$, compared with $58 \mathrm{~nm}$ obtained at $-0.8 \mathrm{~V}$. However, due to time constraints, the sample was not able to be polarized for long enough to observe any aggregation. Despite this, it is clear from the differential capacitance measurements that the highest surface charge, and therefore the best chance of achieving electrostatic stability, is 
obtained at $-1.0 \mathrm{~V}$.

\subsection{SR-SAXS Approach}

The SR-SAXS approach presented in this study was necessary to resolve the moderate particle anisotropy $(\mathrm{AR}<1)$ because of the Bronse-Harte instrument geometry. This geometry is able to resolve lower $q$ values (typically, $q<0.001 \mathrm{~A}^{-1}$ ) compared with a pinhole SAXS setup, but only provides the scattered intensity in one dimension. If it were possible to perform these experiments on a pinhole SAXS setup, multiple sample rotations may not be necessary with such a simple SAS model. Instead, one could use GTSAXS. This approach would be necessary if one were to use a lower flux X-ray source, which requires longer counting times. There are also different possible formulations of the SR-SAXS approach. For example, if the system is sufficiently reversible, it may be possible to make multiple potential sweeps at a single sample angle. However, this approach would require more time and one must be confident that the nucleation and growth kinetics are the same from one sweep to the next.

\section{Conclusions}

For the model $\mathrm{Pb}$ nanoparticle system, complementary suites of SR-SAXS and electrochemical techniques were used to obtain key physical and electrochemical properties of the $\mathrm{Pb}$ nanoparticles. This approach was discussed in detail and provides an experimenter with the ability to obtain: the size and shape of surface bound phases, the volume fraction of the surface scattering phase, as well as the specific differential capacitance of the particle deposit. The proposed approach is valid for systems where growth kinetics are sufficiently slow over the data collection period. In cases where this is may not be true, the faradiaic charge can be used to estimate the effect of growth during data collection and was discussed in detail. With the calibration of the SR-SAXS data, an elevated scattering length density of the surrounding solution was obtained from the model fitting, as reported previously for Ag. This increased scattering length density indicates a high concentration of the dissolved metal precursor exists near the surface, following electrodeposition.

The differential surface capacitance reveals that the optimum potential for electrodeposition of stable $\mathrm{Pb}$ particles is $-1.0 \mathrm{~V}$. Below this overpotential $(-0.8 \mathrm{~V})$, the surface charge on the $\mathrm{Pb}$ particles is insufficient to thwart aggregation, which was observed by SAXS over the 
course of 6 minutes. These aggregates coalesce into moderately oblate features, whose dimensions were resolved by SR-SAXS. These oblate shapes were a consequence of 2-D aggregation and coalescence that was also resolved by SAXS immediately following nucleation. Where the differential capacitance is not constant in the cathodic region, the combined X-ray scattering, electrochemical approach used here can also be used to obtain the optimal particle capacitance to achieve smaller and nanoparticles that have a higher surface charge and therefore more likely to be electrostatically stable.

\section{Acknowledgments}

This research used resources of the Advanced Photon Source, a U.S. Department of Energy (DOE) Office of Science User Facility operated for the DOE Office of Science by Argonne National Laboratory under Contract No. DE-AC02-06CH11357. This study was also partially funded by Lawrence Livermore National Laboratory under Contract No. DE-AC52-07NA27344. The authors would like to thank Dr. Andrew Allen for his very helpful comments and suggestions. 


\section{References}

[1] M.S. Paunovic, Mordechay, Fundamentals of electrochemical deposition, 2nd ed., Wiley, 2006.

[2] A. Abo-Hamad, M. Hayyan, M.A. AlSaadi, M.A. Hashim, Potential applications of deep eutectic solvents in nanotechnology, Chem. Eng. J., 273 (2015) 551-567.

[3] J. Ustarroz, J.A. Hammons, T. Altantzis, A. Hubin, S. Bals, H. Terryn, A Generalized Electrochemical Aggregative Growth Mechanism, J. Am. Chem. Soc., 135 (2013) 11550-11561.

[4] J.L. Fransaer, R.M. Penner, Brownian dynamics simulation of the growth of metal nanocrystal ensembles on electrode surfaces from solution. I. Instantaneous nucleation and diffusion-controlled growth, J. Phys. Chem. B, 103 (1999) $7643-7653$.

[5] H. Liu, R.M. Penner, Size-selective electrodeposition of mesoscale metal particles in the uncoupled limit, J. Phys. Chem. B, 104 (2000) 9131 - 9139.

[6] S. Gorer, R.M. Penner, "Multipulse" electrochemical/chemical synthesis of CdS/S core/shell nanocrystals exhibiting ultranarrow photoluminescence emission lines, J. Phys. Chem. B, 103 (1999) 5750 - 5753.

[7] R.M. Penner, Brownian dynamics simulations of the growth of metal nanocrystal ensembles on electrode surfaces in solution: 2. The effect of deposition rate on particle size dispersion, J. Phys. Chem. B, 105 (2001) $8672-8678$.

[8] Y. Xiong, Y. Xia, Shape-controlled synthesis of metal nanostructures: The case of palladium, Adv. Mater., 19 (2007) 3385-3391.

[9] J. Ustarroz, J.A. Hammons, Y. Van Ingelgem, M. Tzedaki, A. Hubin, H. Terryn, Multipulse electrodeposition of Ag nanoparticles on HOPG monitored by in-situ by Small-Angle X-ray Scattering, Electrochem. Commun., 13 (2011) 1320-1323.

[10] T.J. Woehl, T. Prozorov, The Mechanisms for Nanoparticle Surface Diffusion and Chain Self-Assembly Determined from Real-Time Nanoscale Kinetics in Liquid, J. Phys. Chem. C, 119 (2015) 21261-21269.

[11] M. Jose-Yacaman, C. Gutierrez-Wing, M. Miki, D.Q. Yang, K.N. Piyakis, E. Sacher, Surface diffusion and coalescence of mobile metal nanoparticles, J. Phys. Chem. B, 109 (2005) 9703-9711.

[12] J. Ustarroz, T. Altantzis, J.A. Hammons, A. Hubin, S. Bals, H. Terryn, The Role of Nanocluster Aggregation, Coalescence, and Recrystallization in the Electrochemical Deposition of Platinum Nanostructures, Chem. Mater., 26 (2014) 2396-2406.

[13] L.D. Pachon, G. Rothenberg, Transition-metal nanoparticles: synthesis, stability and the leaching issue, Appl. Organomet. Chem., 22 (2008) 288 - 299.

[14] E.L. Smith, A.P. Abbott, K.S. Ryder, Deep Eutectic Solvents (DESs) and Their Applications, Chem. Rev., 114 (2014) 11060-11082.

[15] Q. Zhang, K. De Oliveira Vigier, S. Royer, F. Jerome, Deep eutectic solvents: syntheses, properties and applications, Chem. Soc. Rev., (2012) 7108 - 7146.

[16] A.P. Abbott, G. Capper, D.L. Davies, H.L. Munro, R.K. Rasheed, V. Tambyrajah, Preparation of novel, moisture-stable, Lewis-acidic ionic liquids containing quaternary ammonium salts with functional side chains, Chem. Commun., (2001) 2010- 2011.

[17] H.-G. Liao, Y.-X. Jiang, Z.-Y. Zhou, S.-P. Chen, S.-G. Sun, Shape-Controlled Synthesis of Gold Nanoparticles in Deep Eutectic Solvents for Studies of Structure-Functionality Relationships in Electrocatalysis, Angew. Chem., Int. Ed., 47 (2008) 9100-9103.

[18] A. Querejeta-Fernandez, J.C. Hernandez-Garrido, H. Yang, Y. Zhou, A. Varela, M. Parras, J.J. Calvino-Gamez, J.M. Gonzalez-Calbet, P.F. Green, N.A. Kotov, Unknown Aspects of Self-Assembly of PbS Microscale Superstructures, Acs Nano, 6 (2012) 3800-3812.

[19] L. Wei, Y.-J. Fan, H.-H. Wang, N. Tian, Z.-Y. Zhou, S.-G. Sun, Electrochemically shape-controlled 
synthesis in deep eutectic solvents of Pt nanoflowers with enhanced activity for ethanol oxidation, Electrochim. Acta, 76 (2012) $468-474$.

[20] J.A. Hammons, T. Muselle, J. Ustarroz, M. Tzedaki, M. Raes, A. Hubin, H. Terryn, Stability, Assembly, and Particle/Solvent Interactions of Pd Nanoparticles Electrodeposited from a Deep Eutectic Solvent, J. Phys. Chem. C, 117 (2013) 14381-14389.

[21] D. Renard, C. McCain, B. Baidoun, A. Bondy, K. Bandyopadhyay, Electrocatalytic properties of in situ-generated palladium nanoparticle assemblies towards oxidation of multi-carbon alcohols and polyalcohols, Colloids Surf., A, 463 (2014) 44-54.

[22] C. Gu, J. Tu, One-Step Fabrication of Nanostructured Ni Film with Lotus Effect from Deep Eutectic Solvent, Langmuir, 27 (2011) 10132 - 10140.

[23] T. Cosgrove, R. Hughes, D. Fermin, J. Riley, J. Eastman, J. Eastoe, B. Vincent, J. Van Duijneveldt, P. Reynolds, N.-O.A. Kwamena, J.P. Reid, R. Richardson, P. Bartlett, S. Davis, W. Briscoe, Colloid Science Principles, methods and applications, A John Wiley and Sons. Ltd, Publication, 2010.

[24] R. Ramachandran, S.-M. Chen, G.G. Kumar, An Overview of Electrochemical Energy Storage Devices of Various Electrodes and Morphological Studies of Supercapacitors, International Journal of Electrochemical Science, 10 (2015) 10355-10388.

[25] J.A. Hammons, J. Ustarroz, T. Muselle, A.A.J. Torriero, H. Terryn, K. Suthar, J. Ilavsky, Supported Silver Nanoparticle and Near-Interface Solution Dynamics in a Deep Eutectic Solvent, The Journal of Physical Chemistry C, 120 (2015) 1534-1545.

[26] A.P. Abbott, J.C. Barron, G. Frisch, S. Gurman, K.S. Ryder, A. Fernando Silva, Double layer effects on metal nucleation in deep eutectic solvents, Phys. Chem. Chem. Phys., 13 (2011) $10224-10231$.

[27] M. Figueiredo, C. Gomes, R. Costa, A. Martins, C.M. Pereira, F. Silva, Differential capacity of a deep eutectic solvent based on choline chloride and glycerol on solid electrodes, Electrochim. Acta, 54 (2009) 2630 2634.

[28] R. Costa, M. Figueiredo, C.M. Pereira, F. Silva, Electrochemical double layer at the interfaces of $\mathrm{Hg} /$ choline chloride based solvents, Electrochim. Acta, 55 (2010) 8916 - 8920.

[29] P. Xiong, J. Zhu, X. Wang, Recent advances on multi-component hybrid nanostructures for electrochemical capacitors, J. Power Sources, 294 (2015) 31-50.

[30] P. Sharma, T.S. Bhatti, A review on electrochemical double-layer capacitors, Energy Convers. Manage., 51 (2010) 2901-2912.

[31] B. Xiong, M. Anouti, Physical Properties of a New Deep Eutectic Solvent Based on a Sulfonium Ionic Liquid as a Suitable Electrolyte for Electric Double-Layer Capacitors, J. Phys. Chem. C, 119 (2015) 970-979. [32] W. Zaidi, A. Boisset, J. Jacquemin, L. Timperman, M. Anouti, Deep Eutectic Solvents Based on N-Methylacetamide and a Lithium Salt as Electrolytes at Elevated Temperature for Activated Carbon-Based Supercapacitors, J. Phys. Chem. C, 118 (2014) 4033-4042.

[33] X. Lu, K.G. Yager, D. Johnston, C.T. Black, B.M. Ocko, Grazing-incidence transmission X-ray scattering: surface scattering in the Born approximation, J. Appl. Crystallogr., 46 (2013) 165-172.

[34] J. Gonzalez-Garcia, J. Iniesta, A. Aldaz, V. Montiel, Effects of ultrasound on the electrodeposition of lead dioxide on glassy carbon electrodes, New J. Chem., 22 (1998) 343-347.

[35] A.I. Bhatt, A.M. Bond, J. Zhang, Electrodeposition of lead on glassy carbon and mercury film electrodes from a distillable room temperature ionic liquid, DIMCARB, J. Solid State Electrochem., 11 (2007) 1593-1603. [36] G.H. Carey, A.L. Abdelhady, Z. Ning, S.M. Thon, O.M. Bakr, E.H. Sargent, Colloidal Quantum Dot Solar Cells, Chem. Rev., 115 (2015) 12732-12763.

[37] R. Suarez, P.K. Nair, Co-deposition of PbS-CuS thin films by chemical bath technique, J. Solid State 
Chem., 123 (1996) 296-300.

[38] J. Ilavsky, A.J. Allen, L.E. Levine, F. Zhang, P.R. Jemian, G.G. Long, High-energy ultra-small-angle X-ray scattering instrument at the Advanced Photon Source, J. Appl. Crystallogr., 45 (2012) 1318-1320.

[39] M. Newville, T. Stensitzki, D.B. Allen, A. Ingargiola, LMFIT: Non-Linear Least-Square Minimization and Curve-Fitting for Python, 2014.

[40] J. Ilavsky, P.R. Jemian, Irena: tool suite for modeling and analysis of small-angle scattering, J. Appl. Crystallogr., 42 (2009) 347 - 353.

[41] F. Lixin, M. Degen, S. Bendle, N. Grupido, J. Ilavsky, The Absolute Calibration of a Small-angle Scattering Instrument with a Laboratory X-ray Source, Journal of Physics: Conference Series, 247 (2010) 1-10.

[42] O. Glatter, O.Kratky, Small Angle X-ray Scattering, Academic Press, 1982.

[43] J.E. Martin, A.J. Hurd, Scattering from Fractals, J. Appl. Crystallogr., 20 (1987) 61-78.

[44] G. Beaucage, Approximations leading to a unified exponential power-law approach to small-angle scattering, J. Appl. Crystallogr., 28 (1995) 717-728.

[45] G. Beaucage, Determination of branch fraction and minimum dimension of mass-fractal aggregates, Phys. Rev. E, 70 (2004).

[46] A. Guinier, G. Fournet, Small-angle scattering of X-rays, Wiley, New York, 1955.

[47] G.J. Brug, A.L.G. Vandeneeden, M. Sluytersrehbach, J.H. Sluyters, The Analysis of Electrode Impedances Complicated By the Presence of A Constant Phase Element, J. Electroanal. Chem., 176 (1984) 275 - 295.

[48] M.E. Orazem, B. Tribollet, Electrochemical Impedance Spectroscopy, John Wiley \& Sons, Inc., 2008.

[49] R. Toniolo, N. Dossi, R. Svigelj, L. Pigani, F. Terzi, O. Abollino, G. Bontempelli, A Deep Eutectic Solvent-based Amperometric Sensor for the Detection of Low Oxygen Contents in Gaseous Atmospheres, Electroanalysis, 28 (2015) 757-763.

[50] A.J. Bard, L.R. Faulkner, Electrochemical Methods Fundamentals and Applications, John Wiley \& Sons, Inc., 2001.

[51] A.J. Bard, M. Stratmann, E.J. Calvo, Encyclopedia of Electrochemistry, Interfacial Kinetics and Mass Transport, Wiley, 2003.

[52] K.E.J. Lehtinen, M.R. Zachariah, Energy accumulation in nanoparticle collision and coalescence processes, J. Aerosol Sci, 33 (2002) 357-368. 


\begin{tabular}{|l|l|l|l|l|l|l|}
\hline Parameter & $-0.9 \mathrm{~V}$ & $\begin{array}{l}-0.9 \mathrm{~V} \\
\text { (with growth) }\end{array}$ & $-1.0 \mathrm{~V}$ & $\begin{array}{l}-1.0 \mathrm{~V} \\
\text { (with growth) }\end{array}$ & $-1.1 \mathrm{~V}$ & $\begin{array}{l}-1.1 \mathrm{~V} \\
\text { (with growth) }\end{array}$ \\
\hline $\begin{array}{l}\bar{R}(\mathrm{~nm}) \pm \\
2 \mathrm{~nm}\end{array}$ & 110.9 & $\begin{array}{l}45^{\circ}=101.3 \\
30^{\circ}=102.0 \\
20^{\circ}=103.1\end{array}$ & 117.6 & $\begin{array}{l}45^{\circ}=109.2 \\
30^{\circ}=109.6 \\
20^{\circ}=110.3\end{array}$ & 122.9 & $45^{\circ}=117.2$ \\
$30^{\circ}=117.6$ \\
$2 \mathrm{~nm}$
\end{tabular}

\section{Table captions}

Table 1 compares the SR-SAXS model fit parameters from the Equation 9 (second, fourth and sixth columns) with those obtained by including particle growth in Equations 19 (third, fifth and seventh columns). Errors associated with all the parameters were obtained from the Jacobian matrix in the least-squares fitting. The errors in parenthesis that are associated with $v$ were taken from the Figure 8 and are larger due to the uncertainty in the phase of the $\mathrm{Pb}$ deposit. 


\section{Figure Captions}

Figure 1 is a plot of the measured potential versus time (red) for one of the potentials (-0.9 V) during the potential sweep procedure described in the Experimental section 2.4. The black triangles are the times at which SAXS data collection finished for each sample angle shown with the blue sample cells. The inset is a simplified schematic showing the scattering vectors used in the sample geometry.

Figure 2 contains three consecutive cyclic voltammograms of the Pb-DES system. The potential was $I_{C} R$ corrected using the mean resistance of $152 \Omega$ obtained from the EIS fitting.

Figure 3 is a log-log plot of the SAXS data collected at sample angles of $45^{\circ}$ (green), $30^{\circ}$ (blue) and $20^{\circ}$ (red). The Unified Equation fits for the non-aggregated particles (black dash) and aggregated particles (black sticks) were used to obtain the mean size of the Pb deposit that aggregate into larger aggregate structures.

Figure 4 is a log-log plot of the background-subtracted SR-SAXS data (right) collected in the potential range where the $\mathrm{Pb}$ deposit can be observed. The data (colored) amd model fits (black) are shown on the log-log plot to the left. The sample cell orientations for each curve are shown in blue.

Figure 5 shows the potential evolution of the particle size during the potential sweep. The evolution in the size distribution, $\sigma$, and aspect ratio are shown in the inset, with very little change over the course of the experiment. The cathodic cycle is shown in blue, while the anodic cycle is shown red. The error bars in Figure 5 were obtained from the jacobian matrix in the least-squares fitting routine.

Figures $6 \mathrm{a}, 6 \mathrm{~b}$ and $6 \mathrm{c}$ are log-log plots showing the difference in model fits with (solid line) and without (dashed line) faradaic growth for the SR-SAXS data collected at $-0.9 \mathrm{~V},-1.0 \mathrm{~V}$, and $-1.1 \mathrm{~V}$, respectively. Each SR-SAXS data set consists of SAXS data collected at $20^{\circ}$ (red) $30^{\circ}$ (blue) and $45^{\circ}$ (green).

Figure 7a and 7b contain the EIS data (markers: square is the modulus and circle is the phase) and models (line) from the cathodic and anodic sweeps, respectively. The selected data from: $0.2 \mathrm{~V}$ (red), $-0.9 \mathrm{~V}$ (green), $-1.0 \mathrm{~V}$ (black) and $-1.1 \mathrm{~V}$ (blue) are shown in each.

Figure 8 is a plot of the differential capacitance obtained from the EIS modeling in the potential range: $0.2 \mathrm{~V}$ to $-1.2 \mathrm{~V}$. The cathodic cycle is shown in blue, while the anodic cycle is shown red. Error bars were obtained from model fitting of the EIS data.

Figure 9 is a plot of the volume fraction obtained from combining the SR-SAXS modeling with the total anodic charge passed at $-0.6 \mathrm{~V}$. Error bars were obtained from the error in the fit parameters obtained by the SR-SAXS modeling, as well as the error associated with the uncertainty in phase (i.e. pure $\mathrm{Pb}$ or pure $\mathrm{PbO}_{2}$ ).

Figure 10 is the differential specific capacitance plot obtained by normalization of the deposit mass. The values in blue represent the cathodic scan, while values in red represent the anodic scan. Error bars in the calculation of $C_{m}$ account for model fitting errors in both the EIS and 
SR-SAXS modeling.

Figure 11 is a log-log plot showing the SAXS data collected at sample angles of $30^{\circ}$ and 4 minutes after electrodeposition at $-0.8 \mathrm{~V}$ (closed squares) and $-1.0 \mathrm{~V}$ (open circles). Comparison of the two data show that smaller particles are obtained at $-1.0 \mathrm{~V}$. 


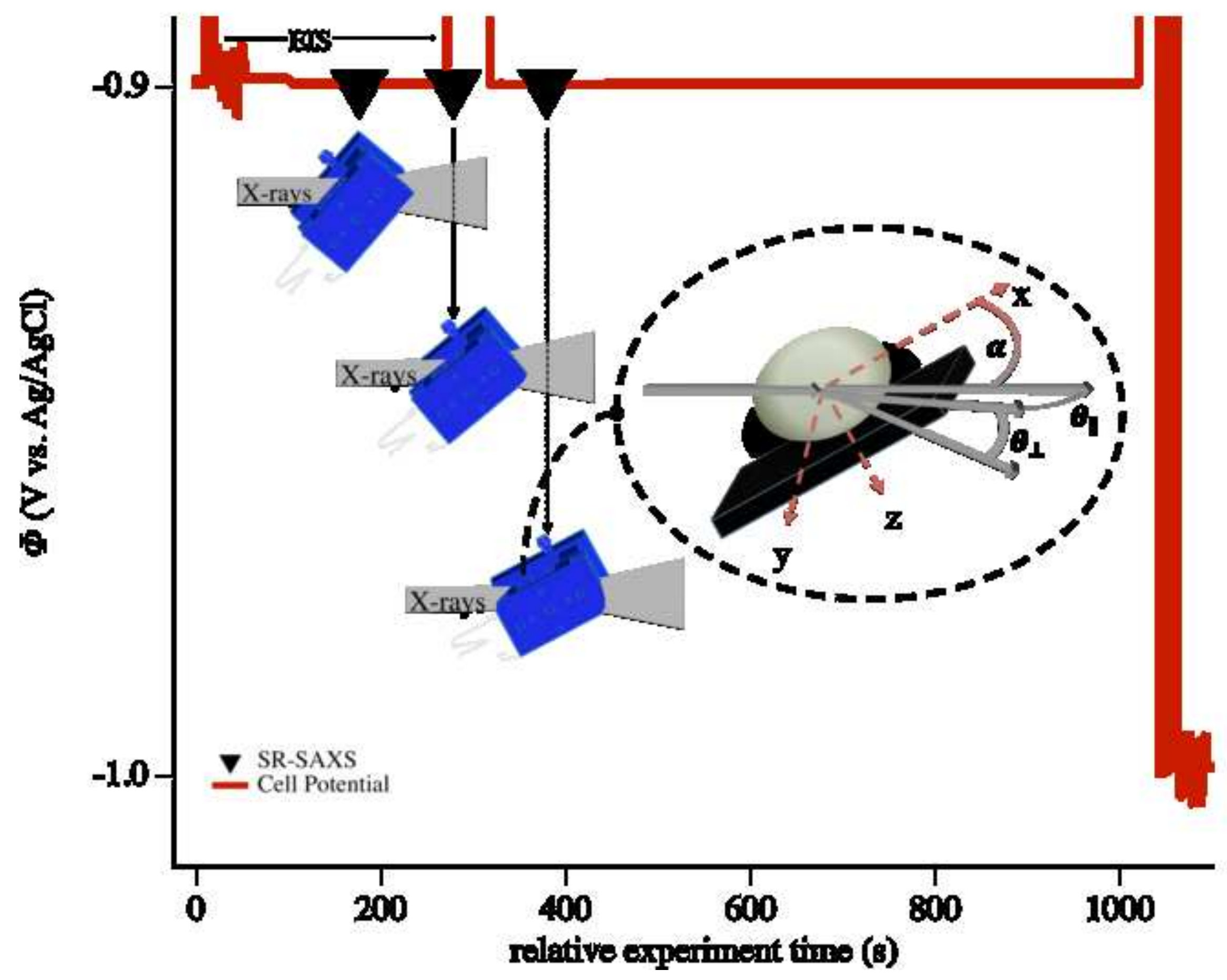




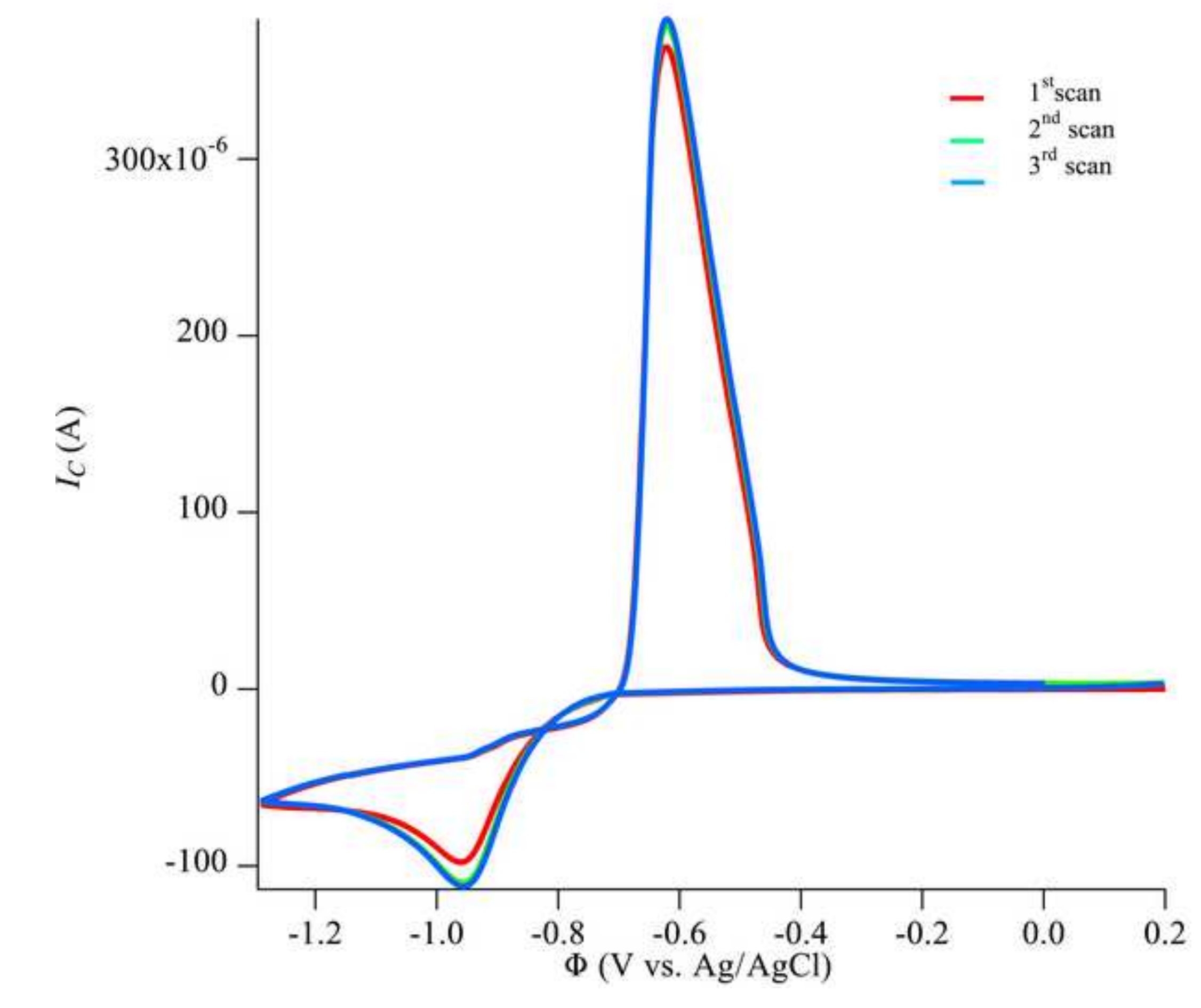




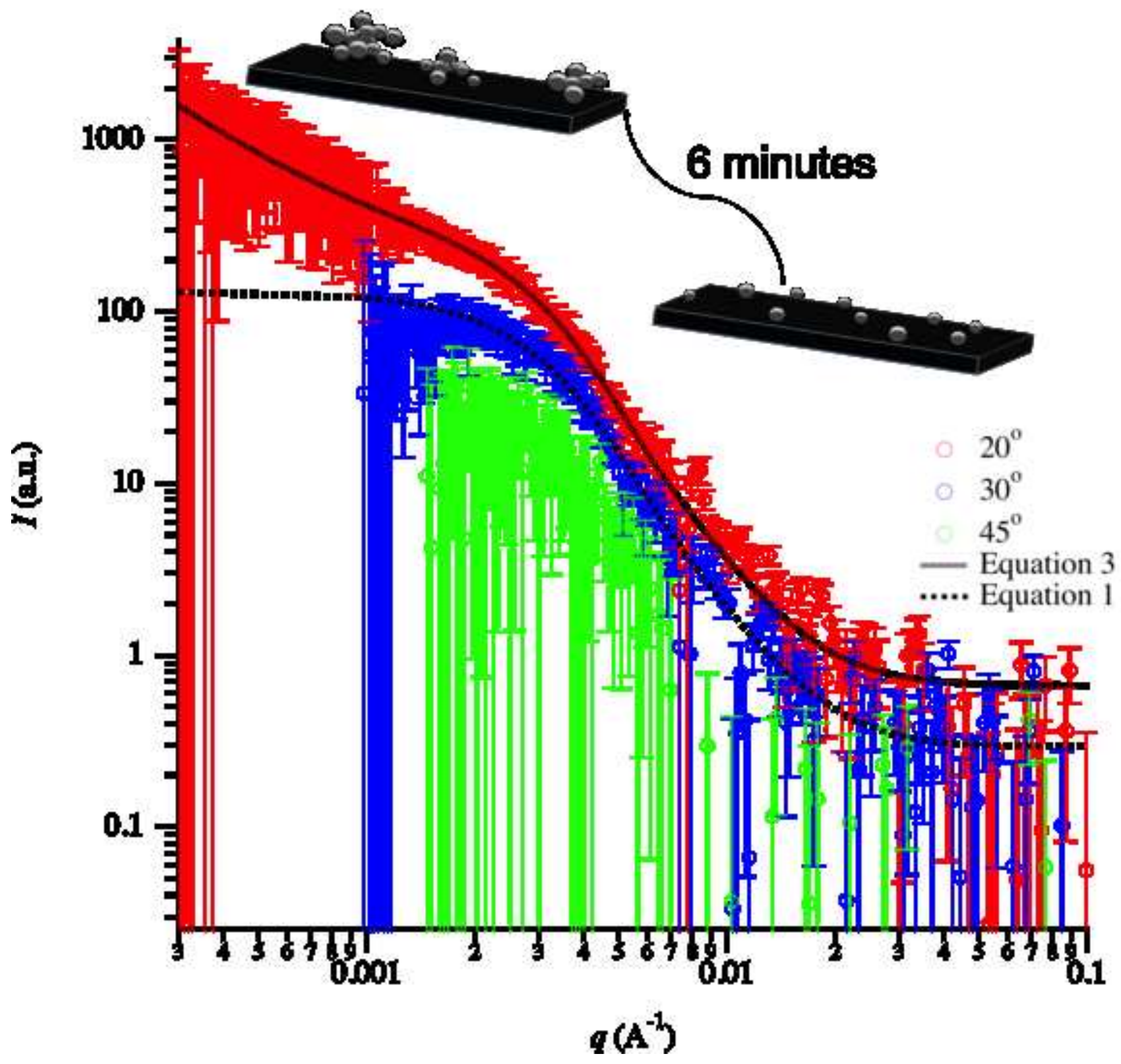




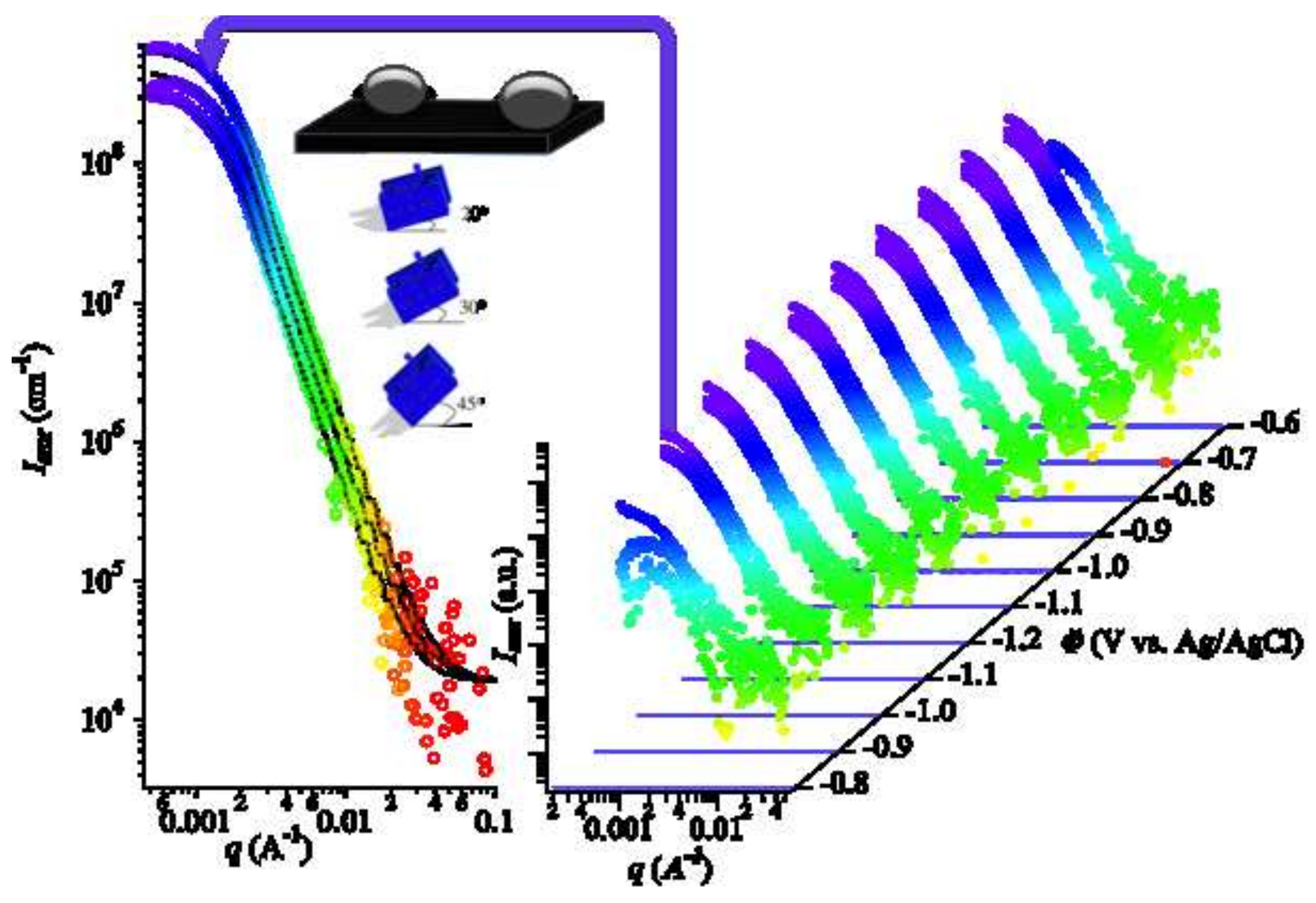




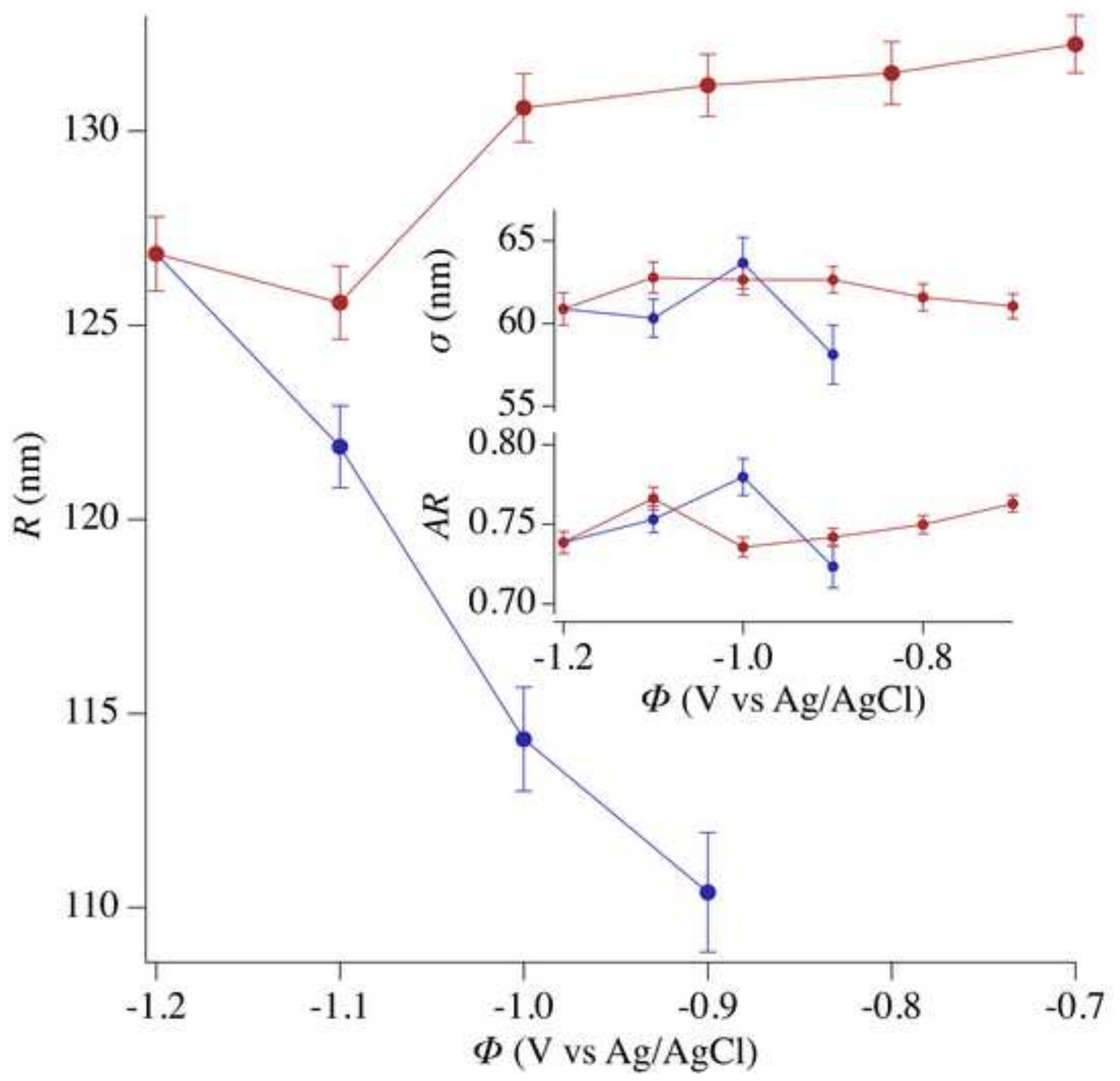



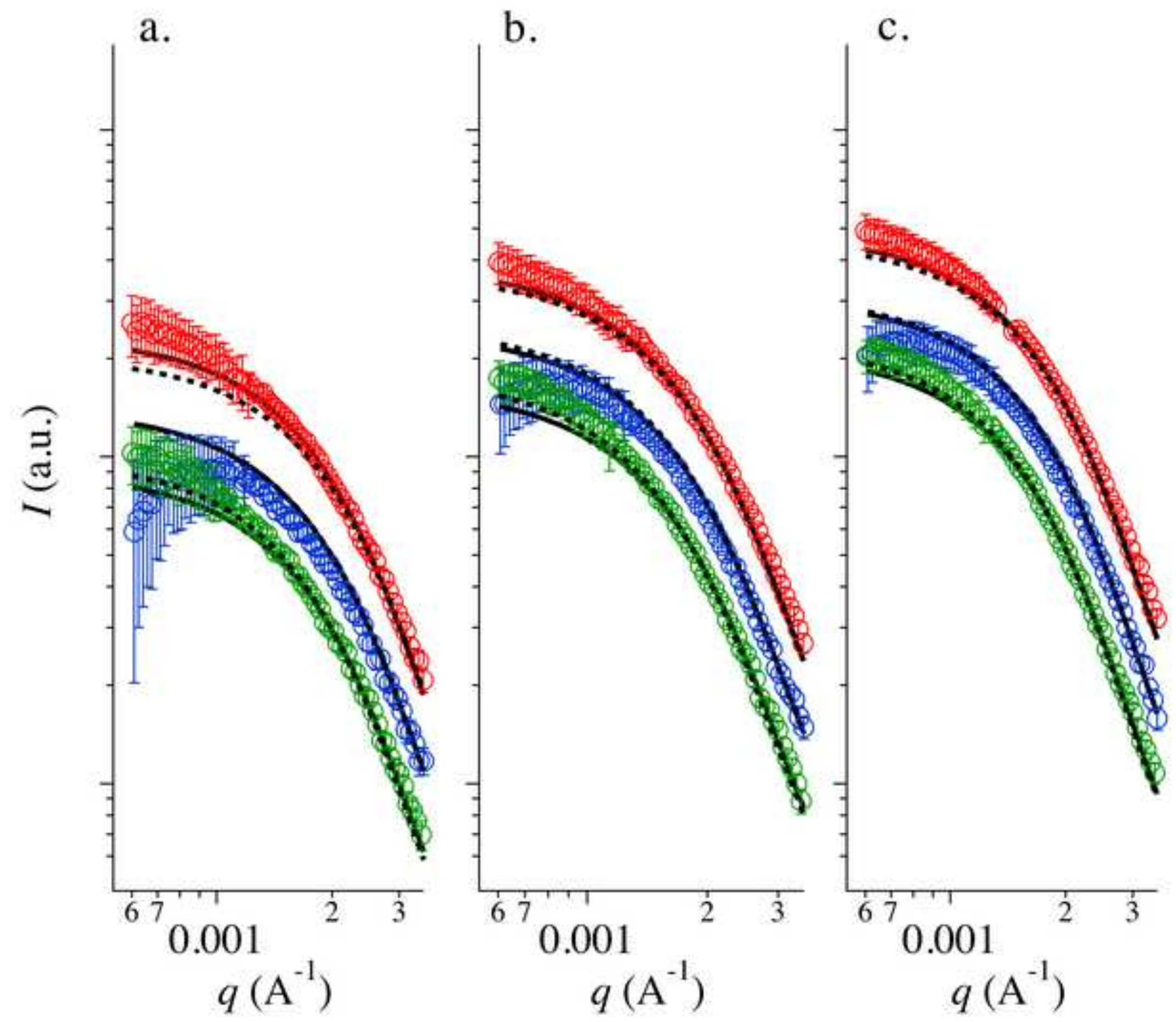


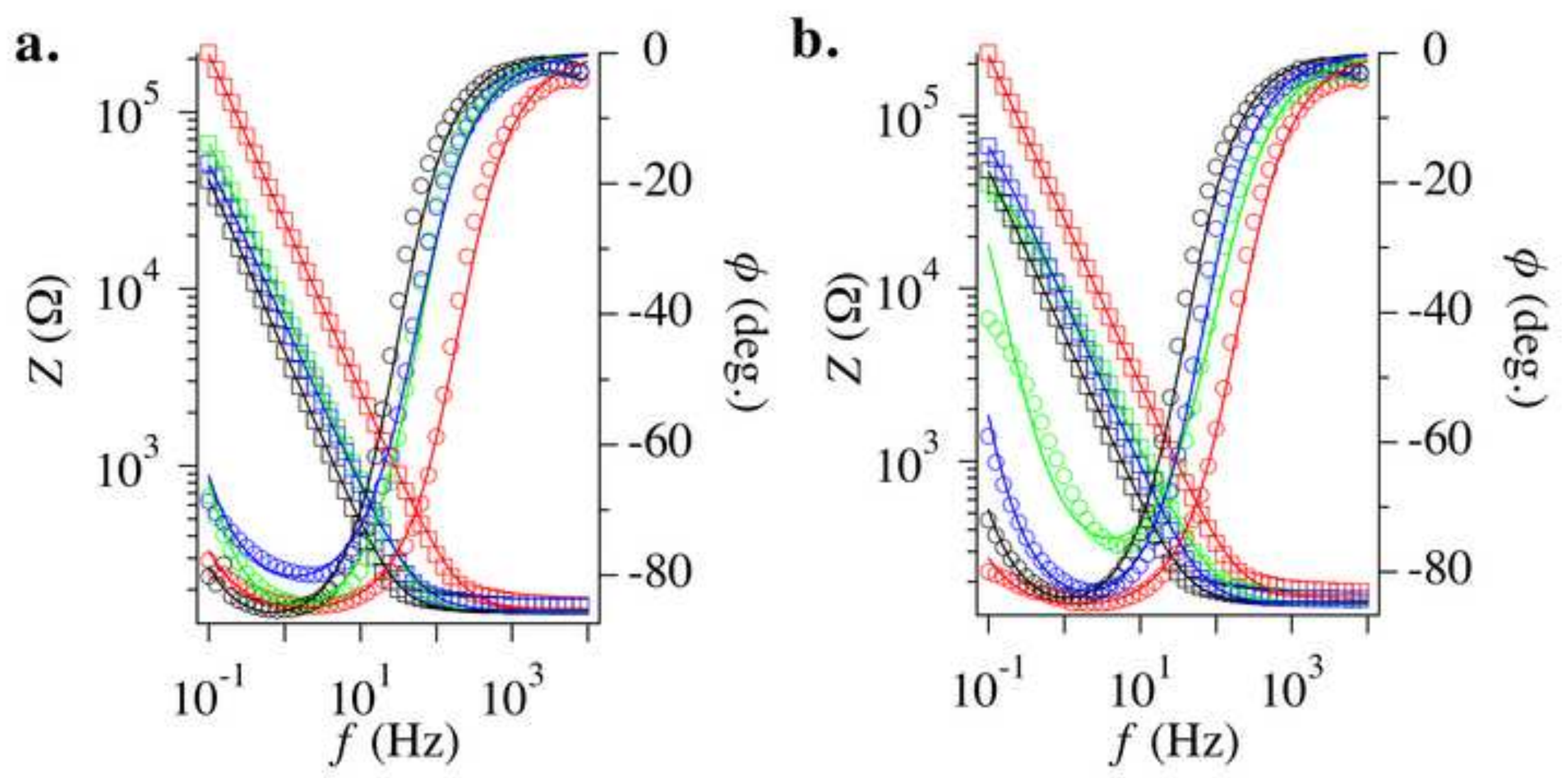




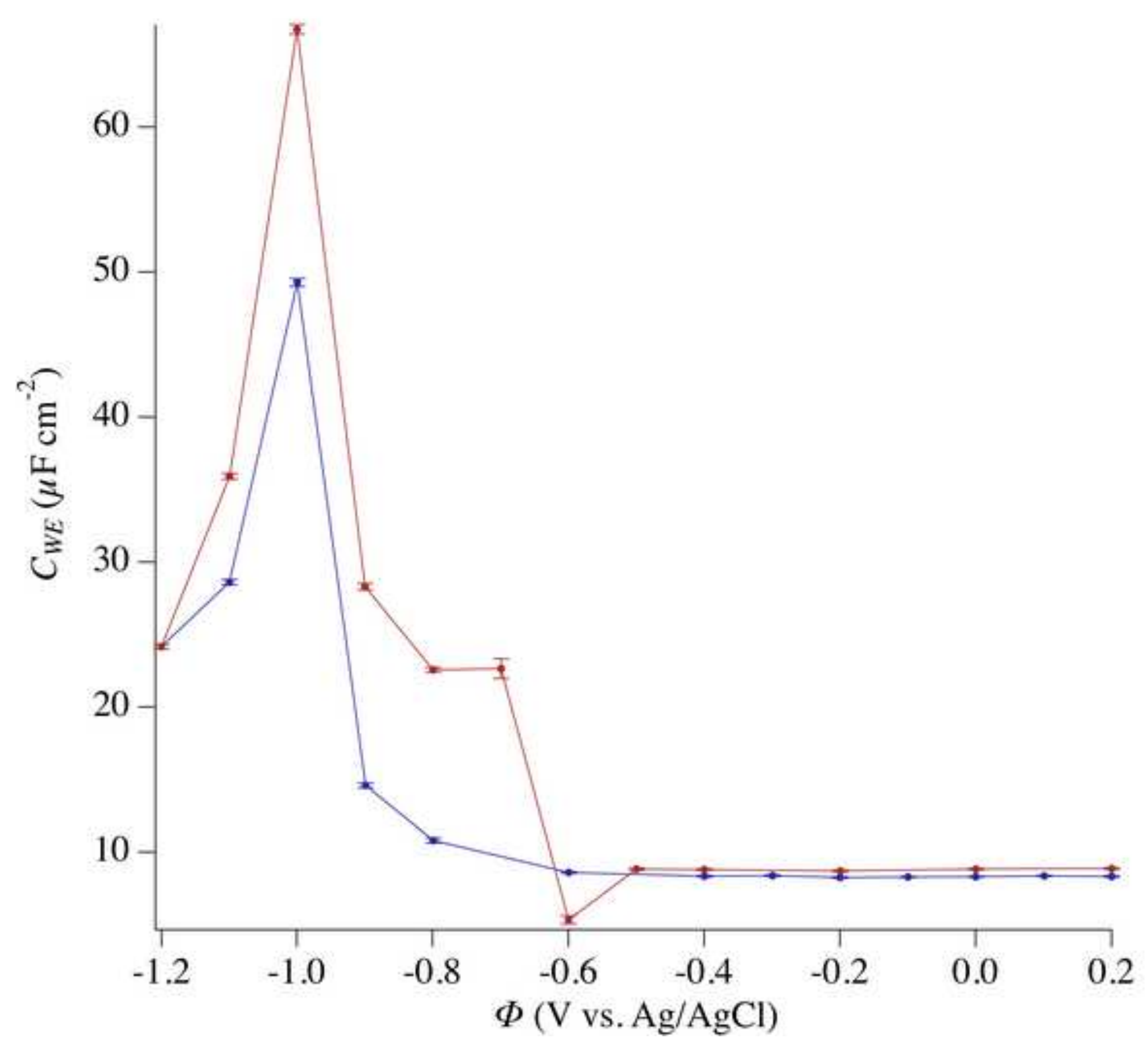




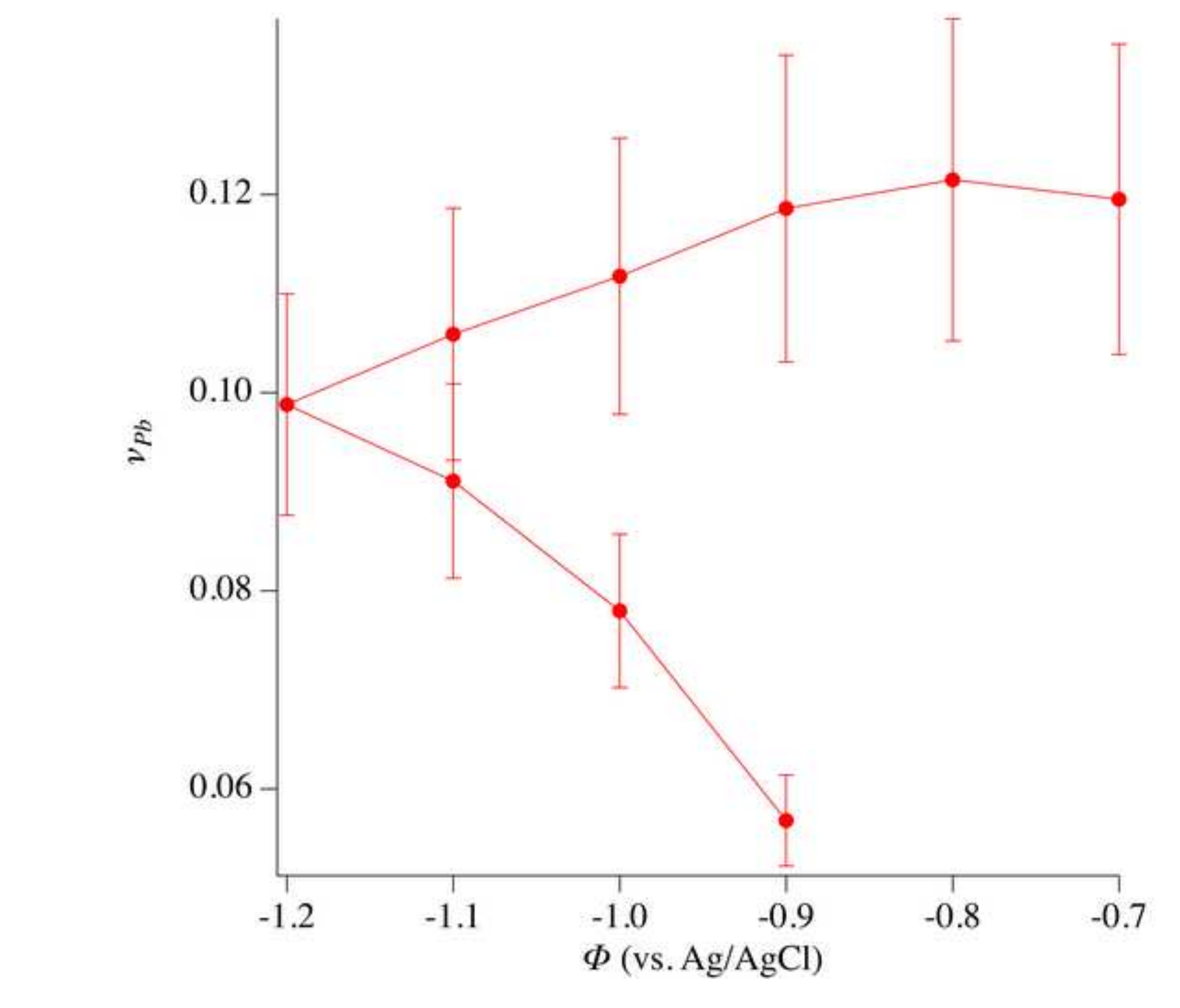




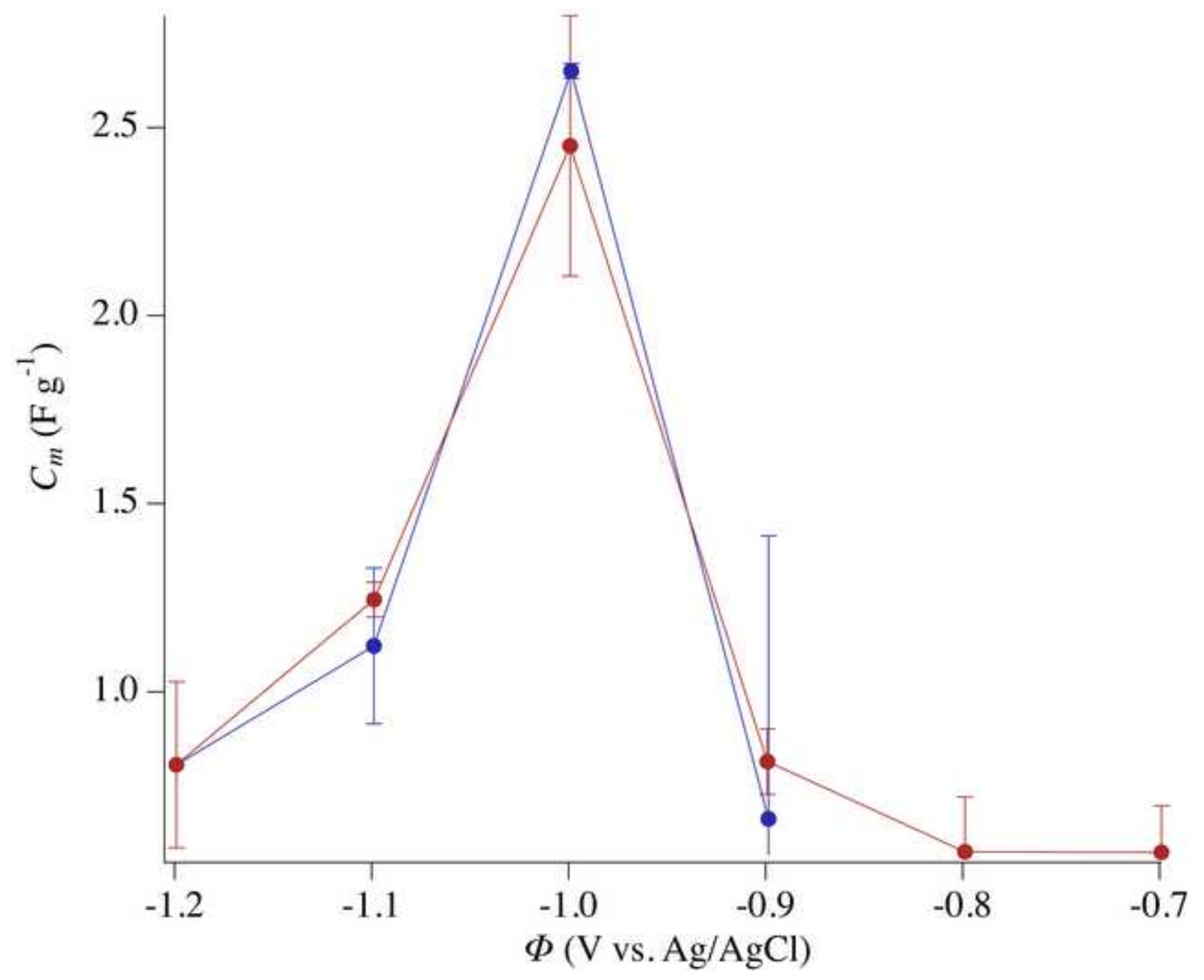




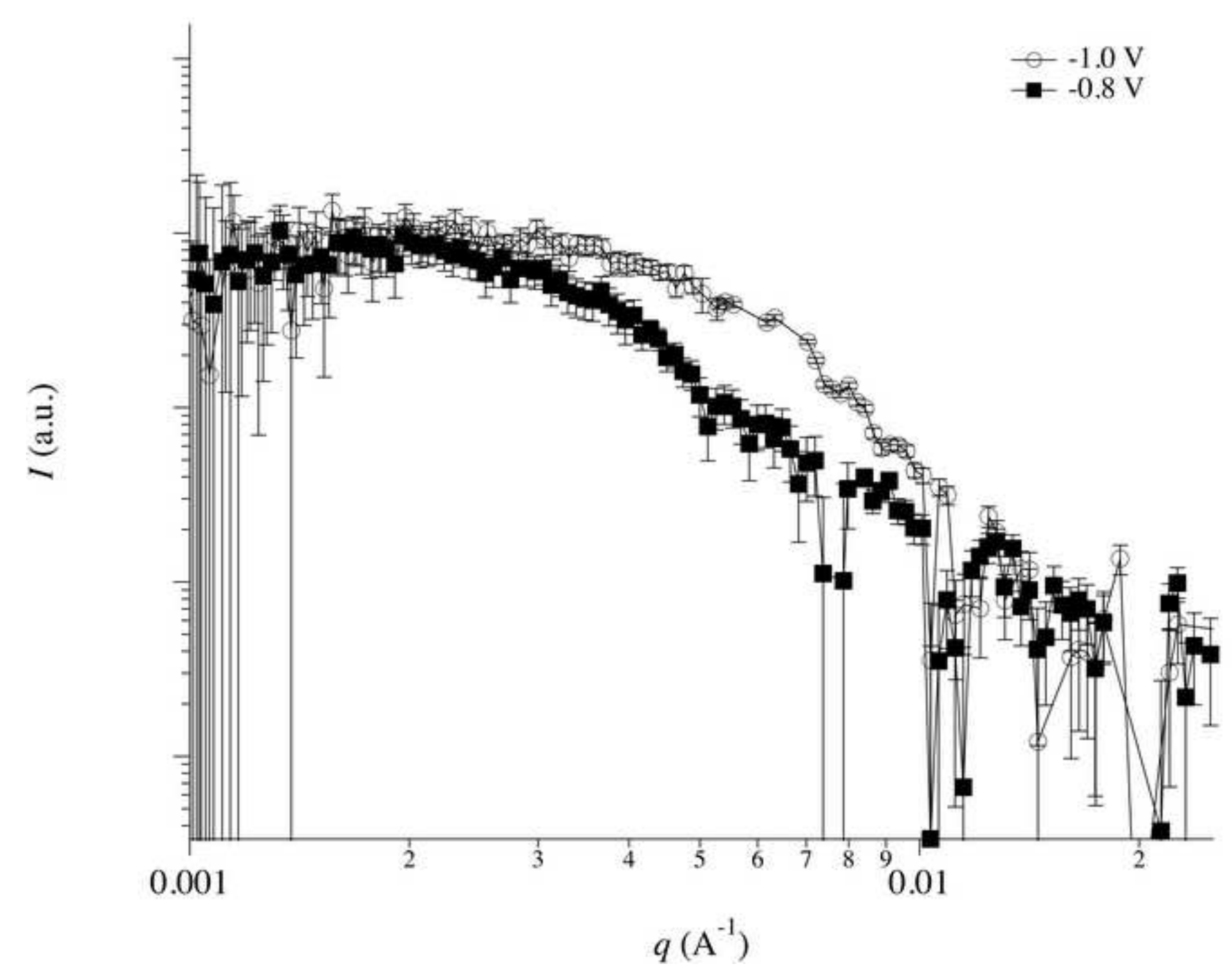

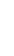

